\title{
SOLUTIONS AND TYPE CURVES OF A FLUID FLOW MODEL FOR NATURALLY FRACTURED RESERVOIRS WITH INFLUX RECHARGE
}

Luis X. Vivas-Cruz, ${ }^{1}$ Jorge Adrián Perera-Burgos, ${ }^{2, *}$
M.A. Taneco-Hernández, ${ }^{3}$ E Alfredo González-Calderón ${ }^{4}$

${ }^{1}$ Centro de Ingeniería y Desarrollo Industrial (CIDESI), Av. Playa Pie de la Cuesta 702, Desarrollo San Pablo, Querétaro, Qro, 76125, México

${ }^{2}$ CONACYT - Unidad de Ciencias del Agua, Centro de Investigación Científica de Yucatán A.C., Calle 8, No. 39, Mz. 29, S.M. 64, Cancún, Quintana Roo, 77524, México

${ }^{3}$ Facultad de Matemáticas, Universidad Autónoma de Guerrero, Av. Lázaro Cárdenas, Cd. Universitaria Sur, Chilpancingo, Guerrero, 39087, México

${ }^{4}$ CONACYT - CIDESI sede Campeche, Ctra. Carmen Puerto Real km 7.5, Mundo Maya, Cd. del Carmen, Campeche 24153, México

*Address all correspondence to: Jorge Adrián Perera-Burgos, CONACYT - Unidad de Ciencias del Agua, Centro de Investigación Científica de Yucatán A.C., Calle 8, No. 39, Mz. 29, S.M. 64, Cancún, Quintana Roo, 77524, México, E-mail: jorge.perera@cicy.mx

Original Manuscript Submitted: 2/17/2020; Final Draft Received: 8/23/2020

Modeling of fluid flow considering radially symmetric reservoirs is common in groundwater science and petroleum engineering. The Hankel transform is suitable for solving boundary value problems, considering this flow geometry. However, there are few applications of this transform for reservoirs with a finite wellbore radius, although there are formulas of the finite Hankel transform for homogeneous boundary conditions. In this work, we refer to them as the Cinelli formulas, which are used to obtain novel solutions for transient fluid flow in bounded naturally fractured reservoirs with time-varying influx at the outer boundary, i.e., a technique to incorporate inhomogeneous boundary conditions based on the Cinelli formulas is developed. An analysis shows that the results of the solutions are highly oscillating and slowly convergent. Nevertheless, we show that this problem is largely overcome when the long-time solution is expressed as a closed relationship. Accordingly, we present the characteristic drawdown pressure curves and its Bourdet derivatives for a double-porosity reservoir with influx recharge. These curves allow us to distinguish between the pressure drops of a single-porosity reservoir with influx recharge from that of a double-porosity closed reservoir, which have been stated in the literature to resemble one another. Similarly, double-and triple-porosity reservoirs are analyzed.

KEY WORDS: fluid flow model, naturally fractured reservoir, influx at the outer boundary, joint LaplaceHankel transform

\section{INTRODUCTION}

In groundwater science and petroleum engineering, the modeling of fluid flow in underground reservoirs has impact on project planning and reserve estimates. However, current models for fluid flow in reservoirs have limitations that affect their accuracy when they are applied in the tasks just mentioned. Therefore, there is a need for including in the governing equations natural properties such as storage, porosities, permeabilities, wellbore storage, skin factor, or recharge. Furthermore, new mathematical developments with applications in pumping or well tests (Chen, 1990; De Smedt, 2011; Ju, 2014; Liu and Chen, 1990; Ozkan and Raghavan, 1988; Wu, 2002; Young, 1992) allow us to 
understand phenomena, which could be challenging otherwise (Cai et al., 2016; Da Prat, 1990; Kuhlman et al., 2015; Nie et al., 2012; Singhal and Gupta, 2010; Zhou et al., 2019).

Many fluid flow models have exact solutions in Laplace space, but their inverse transforms can be quite complex to obtain by means of contour integration in the complex plane (González-Calderón et al., 2017; Yao et al., 2012). Remarkably, the Hankel transform provides a simple way to treat radially symmetric problems, since their inverse transform formulas are the solutions of the models (Cinelli, 1965; Jiang and Gao, 2010; Sneddon, 1946). One of these solutions is given by Cinelli (1965); nevertheless, because their relationships are for homogeneous boundary conditions, they are strongly limited in their application to describe fluid flow in reservoirs associated with a hollow disk geometry. In order to extend the applicability of the finite Hankel transform derived by Cinelli, Xi and Yuning (1991) and Wang and Gong (1992) developed a mathematical procedure that can be used for inhomogeneous boundary conditions. In those studies the initial and boundary value problems are expressed as the sum of a dynamic part with inhomogeneous boundary conditions and a quasi-static part with homogeneous boundary conditions in such a way that the solution from the quasi-static differential equation can make use of the mentioned formulas. In a similar fashion, in this work we divided the solution into a stationary and a transient part, which appropriately allows us to solve the problem with inhomogeneous conditions. By contrast, Xi and Yuning (1991) and Wang and Gong (1992) solved a hyperbolic model for describing displacement in elastodynamics, while in this study we solve a parabolic model for describing the fluid flow in a double-porosity reservoir. Models of fluid flow in reservoirs with inhomogeneous boundary conditions, for example, influx recharge, have been the subject of different studies (del Angel et al., 2014; Doublet and Blasingame, 1995; Wang et al., 2017), but, to our best knowledge they have not been analytically solved for double-porosity systems.

In addition to the Hankel transform, in order to solve partial differential equations, the Laplace transforms can be jointly used, taking us to the joint Laplace-Hankel transform, or JLHT (Debnath and Bhatta, 2014; Poularikas, 2010). An application in models of fluid flow in reservoirs is found in Babak and Azaiez (2014), where finite and infinite reservoirs are considered, each of them having a centered well with an infinitely small radius. For hollow-disk geometry, the finite Hankel transform was used to solve a triple-porosity fluid flow model with a constant pressure and zero flux at the inner and outer boundaries, respectively, and considering a nonzero well radius (Clossman, 1975). Also, the JLHT has been used in the study of crossflow in stratified systems; for instance, Boulton and Streltsova (1977) provided the relationships of flow through horizontal layers of a fissured aquifer, restricted to have vertical permeability, and with a wellbore represented as a line source pumping at a constant rate. A similar system with a partially penetrating well is found in a study by Javandel and Witherspoon (1983). In the early 60s, Katz and Tek (1962) and Russell and Prats (1962) were the pioneers in the study of crossflow in stratified reservoirs using the Fourier and the Hankel transforms, respectively. Subsequently, Prats (1986) showed that stratified reservoirs and single-layer reservoirs have similar behavior for large periods of time. More complex systems were analyzed by Shah and Thambynayagam (1992): they included two flowing intervals in a partial completion well. On the other hand, exact solutions by means of other mathematical procedures can be consulted in Gomes and Ambastha (1993) and Ehlig-Economides and Joseph (1987), for layered aquifers, and in Chen (1989) and Matthews and Russell (1967), for oil reservoirs. Additional applications of JLHT are found in Carslaw and Jaeger (1959), Debnath and Bhatta (2014), and Poularikas (2010).

There is a lack of exact solutions using the JLHT for models of fluid flow in bounded or infinite reservoirs. Partly, this may be due to the complexities inherent to the method that will be exposed in this work. In order to contribute to the studies in this direction, we use the JLHT to solve the double-porosity model of Warren and Root (1963) using the following combinations of specific boundary conditions (BCs): Dirichlet-Dirichlet (DD), DirichletNeumann (DN), Neumann-Dirichlet (ND), and Neumann-Neumann (NN). The inner condition is given by either a constant terminal pressure or a constant terminal rate. Meanwhile, the outer boundary has a constant pressure or has an influx recharge defined as a ramp rate function to simulate natural water influx or slow-starting waterfloods from injector wells (Doublet and Blasingame, 1995). Also, this latter function can be interpreted as rock heterogeneities at the outer boundary that obstruct the flow channels (del Angel et al., 2014; Doublet and Blasingame, 1995). We note that our solutions generalize the relationships of fluid flow in a single-porosity medium, which were released in other works and can be found in Muskat (1934), for DD-BCs; Hurst (1934) and Muskat (1934), for DN-BCs; Matthews and Russell (1967), for ND-BCs; and in del Angel et al. (2014); Matthews and Russell (1967); Muskat (1934), for 
NN-BCs. Lu et al. (2018, 2019a) give alternative solution methods for fluid flow in a circular or rectangular closedboundary reservoir, respectively.

The Warren and Root model has been widely used in well and pumping test analysis (Ahmed and McKinney, 2011; Bourdet et al., 1989; Gringarten, 2008; Kruseman et al., 1994). Nevertheless, the NN-BCs case deserves special attention, due to the lack of studies in this regard, in such a way that it is important to provide type curves that include the effects of influx recharge at the outer boundary. Indeed, it has been observed that a single-porosity reservoir with influx recharge has a characteristic behavior similar to that of a double-porosity reservoir without influx recharge (del Angel et al., 2014; Doublet and Blasingame, 1995). By extension, it could be expected that a double-porosity reservoir with influx recharge has a behavior similar to that given by a triple-porosity reservoir without influx recharge. This observation is attended in this work in order to elucidate whether the drawdown pressures of models with recharge and without it can be considered equivalent.

The contribution of this work is threefold:

1. Obtain analytical solutions for the aforementioned study cases.

2. Present characteristic behaviors of the drawdown pressure and flux for the study cases.

3. Show the similarities and differences between a model with influx recharge and without it, but this latter case with an additional porosity.

This work is organized as follows: In Sections 2 and 3, the flow model and the boundary conditions are presented, respectively. In Section 4, the procedure to find the exact solutions is given. Subsequently, in Section 5, a numerical validation using the Stehfest method is carried out, the convergence of the Cinelli solutions to the exact result is analyzed, and a discussion about the stationary solutions is also presented. In Section 6, the characteristic curves of the fluid flow model are exposed, and a comparison is done between models with influx recharge and without it. Finally, in Section 7, some general conclusions are drawn.

\section{FLOW MODEL}

Radial fluid flow in a double-porosity medium is described by the model of Warren and Root (1963). This model considers a slightly compressible fluid through two overlapping porous systems: matrix and fractures. The matrix discharges into the fractures, and the fractures carry the flow toward the wellbore. The matrix has a low permeability and a high storage capacity, while the fracture system has a high permeability and a low storage capacity. It is also assumed to be a homogeneous and isotropic porous system. An illustration of the reservoir and its fluid flow is given in Fig. 1.

The Warren and Root model considers an equation for the pressure $h_{2}$ in fractures,

$$
\frac{k_{2}}{\mu} \frac{1}{r} \frac{\partial}{\partial r}\left(r \frac{\partial h_{2}}{\partial r}\right)-\phi_{1} c_{1} \frac{\partial h_{1}}{\partial t}=\phi_{2} c_{2} \frac{\partial h_{2}}{\partial t},
$$

and an equation for the pressure $h_{1}$ in matrix blocks,

$$
\phi_{1} c_{1} \frac{\partial h_{1}}{\partial t}=\frac{\alpha \kappa_{1}}{\mu}\left(h_{2}-h_{1}\right) .
$$

In the previous equations, subscripts 1 and 2 indicate the matrix medium and the fracture medium, respectively; $\kappa_{i}, \phi_{i}$, and $c_{i}$, are the permeabilities, porosities, and total compressibilities of medium $i$; $\mu$ is the fluid viscosity; and $\alpha$ is the shape factor.

Equations (1) and (2) in reduced units are as follows:

$$
\begin{array}{rlrl}
\omega \frac{\partial h_{2 D}}{\partial t_{D}}+(1-\omega) \frac{\partial h_{1 D}}{\partial t_{D}} & =\frac{\partial^{2} h_{2 D}}{\partial r_{D}^{2}}+\frac{1}{r_{D}} \frac{\partial h_{2 D}}{\partial r_{D}}, & & 1 \leq r_{D} \leq r_{D \text { ext }}, \\
(1-\omega) \frac{\partial h_{1 D}}{\partial t_{D}} & =\lambda\left(h_{2 D}-h_{1 D}\right), & t_{D} \geq 0,
\end{array}
$$

Volume 23, Issue 10, 2020 


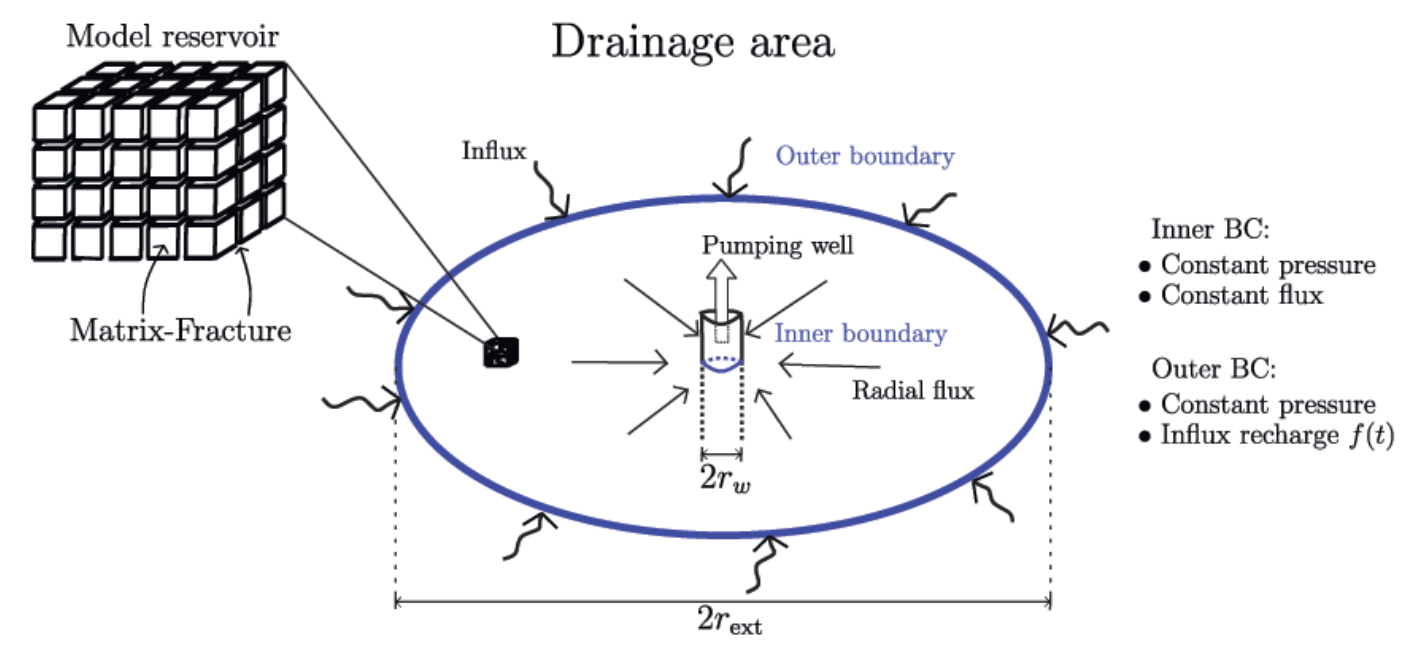

FIG. 1: Schematic representation of the reservoir and the fluid flow. Drainage area of reservoir is illustrated by a big circle of radius $r_{\text {ext }}$, while in its center there is a cylinder of radius $r_{w}$ that represents the pumping well. Other boundary conditions used in this work are specified on the right of the figure.

where the dimensionless dependent variables are given by

$$
h_{i D} \equiv \begin{cases}\frac{h_{0}-h_{i}(r, t)}{h_{0}-h_{w}}, & \text { for constant pressure } \\ \frac{2 \pi H \kappa_{2}}{\mu q}\left[h_{0}-h_{i}(r, t)\right], & \text { for constant flux. }\end{cases}
$$

The dimensionless independent variables are defined as

$$
t_{D} \equiv \frac{\kappa_{2} t}{\mu r_{w}^{2}\left(\phi_{1} c_{1}+\phi_{2} c_{2}\right)}, \quad r_{D} \equiv \frac{r}{r_{w}}
$$

and the parameters of the model are

$$
\omega \equiv \frac{\phi_{2} c_{2}}{\phi_{1} c_{1}+\phi_{2} c_{2}}, \quad \lambda \equiv \frac{\alpha r_{w}^{2} \kappa_{1}}{\kappa_{2}}, \quad r_{D \mathrm{ext}} \equiv \frac{r_{\mathrm{ext}}}{r_{w}} .
$$

In these latter definitions, $r_{w}$ is the well radius; $r_{D \text { ext }}$ is the dimensionless outer radius; $\omega$ is the fracture storage coefficient; $\lambda$ is the interporosity flow coefficient; $h_{0}$ and $h_{w}$ are the reference pressure and the pressure at the bottomhole, respectively; $H$ is the thickness of the uniform horizontal formation; and $q$ is the constant volumetric flow rate.

In addition, the flux $j_{2}$ in reduced units is defined as follows (Matthews and Russell, 1967):

$$
j_{2 D}\left(t_{D}\right)=-\left.r_{D} \frac{\partial h_{2 D}\left(r_{D}, t_{D}\right)}{\partial r_{D}}\right|_{r_{D}=1},
$$

where

$$
j_{2 D}= \begin{cases}\frac{\mu}{2 \pi H \kappa_{2}\left(h_{w}-h_{0}\right)} j_{2}, & \text { for constant pressure } \\ -\frac{j_{2}}{q}, & \text { for constant flux. }\end{cases}
$$




\section{INITIAL AND BOUNDARY CONDITIONS}

Assuming that the reservoir has a constant pressure at time zero, the initial conditions are written as

$$
h_{1 D}\left(r_{D}, 0\right)=h_{2 D}\left(r_{D}, 0\right)=0, \quad 1 \leq r_{D} \leq r_{D \text { ext }}
$$

The boundary condition at the bottomhole, when a constant pressure is imposed, is given by

$$
h_{2 D}\left(1, t_{D}\right)=1, \quad t_{D} \geq 0
$$

On the other hand, when a constant flow at the bottomhole is imposed and an influx recharging the reservoir through the outer boundary is considered, the boundary conditions are as follows:

$$
r_{D} \frac{\partial h_{2 D}\left(r_{D}, t_{D}\right)}{\partial r_{D}}= \begin{cases}-1, & \text { for } r_{D}=1, t_{D} \geq 0, \\ f\left(t_{D}\right)=-q_{\text {ext }}\left(1-\mathrm{e}^{-t_{D} / \gamma}\right), & \text { for } r_{D}=r_{D \text { ext }}, t_{D} \geq 0,\end{cases}
$$

where $q_{\mathrm{ext}} \geq 0$ is the influx factor, and $\gamma$ is a parameter to change the slope of the ramp rate function (del Angel et al., 2014; Doublet and Blasingame, 1995). Note that $q_{\mathrm{ext}}=0$ is for a reservoir with zero flux at the outer boundary (van Everdingen and Hurst, 1949).

A constant pressure at the outer boundary is also considered:

$$
h_{2 D}\left(r_{D \text { ext }}, t_{D}\right)=0, \quad t_{D} \geq 0 \text {. }
$$

Some combinations of these BCs are shown in Table 1. They comprise the case studies analyzed in this work.

\section{INTEGRAL TRANSFORM SOLUTIONS}

In this section, using the JLHT and its inversion formulas, solutions of fluid flow in a double-porosity medium [Eq. (3)] for the initial-boundary conditions in Section 3 are presented. To validate the solutions, comparisons with results from the Stehfest method are made in Section 5. Details of the calculations can be found in Appendix A. Henceforth, for simplicity in notation, we omit the subscript $D$ of the dimensionless variables previously defined.

Equation (3) in Laplace space is written as

$$
\begin{aligned}
\omega s \widehat{h}_{2}+ & (1-\omega) s \widehat{h}_{1}=\frac{\partial^{2} \widehat{h}_{2}}{\partial r^{2}}+\frac{1}{r} \frac{\partial \widehat{h}_{2}}{\partial r}, \quad 1 \leq r \leq r_{\mathrm{ext}}, \\
(1-\omega) s \widehat{h}_{1} & =\lambda\left(\widehat{h}_{2}-\widehat{h}_{1}\right)
\end{aligned}
$$

TABLE 1: Case studies and their dimensionless boundary conditions used to solve the model by Eq. (3)

\begin{tabular}{|c|c|c|c|}
\hline Case & Boundary conditions & Inner boundary & Outer boundary \\
\hline DD-BCs & Dirichlet-Dirichlet & $h_{2 D}\left(1, t_{D}\right)=1$ & $h_{2 D}\left(r_{D \text { ext }}, t_{D}\right)=0$ \\
DN-BCs & Dirichlet-Neumann & $h_{2 D}\left(1, t_{D}\right)=1$ & $\left.r_{D} \frac{\partial h_{2 D}\left(r_{D}, t_{D}\right)}{\partial r_{D}}\right|_{r_{D}=r_{D \text { ext }}}=f\left(t_{D}\right)$ \\
ND-BCs & Neumann-Dirichlet & $\left.r_{D} \frac{\partial h_{2 D}\left(r_{D}, t_{D}\right)}{\partial r_{D}}\right|_{r_{D}=1}=-1$ & $h_{2 D}\left(r_{D \text { ext }}, t_{D}\right)=0$ \\
NN-BCs & Neumann-Neumann & $\left.r_{D} \frac{\partial h_{2 D}\left(r_{D}, t_{D}\right)}{\partial r_{D}}\right|_{r_{D}=1}=-1$ & $\left.r_{D} \frac{\partial h_{2 D}\left(r_{D}, t_{D}\right)}{\partial r_{D}}\right|_{r_{D}=r_{D \mathrm{ext}}}=f\left(t_{D}\right)$ \\
\hline
\end{tabular}


and the BCs in Table 1 are summarized as follows:

$$
\begin{gathered}
\widehat{h}_{2}(1, s)=\frac{1}{s}, \\
\left.r \frac{\partial \widehat{h}_{2}(r, s)}{\partial r}\right|_{r=1}=-\frac{1}{s}, \\
\widehat{h}_{2}\left(r_{\mathrm{ext}}, s\right)=0, \\
\left.r \frac{\partial \widehat{h}_{2}(r, s)}{\partial r}\right|_{r=r_{\mathrm{ext}}}=\widehat{f}(s), \\
\widehat{h}_{i}(r, s)=0, \quad i=1,2,
\end{gathered}
$$

where $s$ is the Laplace transform variable, $\widehat{x}$ denotes the Laplace transform of $x$, and

$$
\widehat{f}(s)=-q_{\mathrm{ext}}\left(\frac{1}{s}-\frac{1}{s+1 / \gamma}\right) .
$$

From Eq. (14), the Laplace transform of pressure in the fractures obeys the modified Bessel ordinary differential equation:

$$
\frac{d^{2} \widehat{h}_{2}}{d r^{2}}+\frac{1}{r} \frac{\widehat{d h}_{2}}{d r}-\eta(s) \widehat{h}_{2}=0, \quad 1 \leq r \leq r_{\mathrm{ext}}
$$

where

$$
\eta(s)=\frac{s \omega(1-\omega)+\lambda}{s(1-\omega)+\lambda} s .
$$

We use the finite Hankel transform to obtain an analytical solution of our study model, so taking this transform to Eq. (21) leads to the following expressions in the joint Laplace-Hankel space:

$$
\begin{aligned}
\tilde{\widehat{h}}_{2}\left(k_{i}, s\right) & =\mathcal{F}\left(k_{i}, s\right)\left(\left.\widehat{h}_{2}(r, s)\right|_{r=r_{\mathrm{ext}}} \frac{\left.J_{0}\left(r k_{i}\right)\right|_{r=1}}{\left.J_{0}\left(r k_{i}\right)\right|_{r=r_{\mathrm{ext}}}}-\left.\widehat{h}_{2}(r, s)\right|_{r=1}\right), \quad \text { for DD-BCs, } \\
& =\mathcal{F}\left(k_{i}, s\right)\left(\left.r \frac{\partial \widehat{h}_{2}}{\partial r}\right|_{r=r_{\mathrm{ext}}} \frac{\left.J_{0}\left(r k_{i}\right)\right|_{r=1}}{\left.k_{i} J_{0}^{\prime}\left(r k_{i}\right)\right|_{r=r_{\mathrm{ext}}}}-\left.\widehat{h}_{2}(r, s)\right|_{r=1}\right), \quad \text { for DN-BCs, } \\
& =\mathcal{F}\left(k_{i}, s\right)\left(\left.\widehat{h}_{2}(r, s)\right|_{r_{\mathrm{ext}}} \frac{\left.J_{0}^{\prime}\left(r k_{i}\right)\right|_{r=1}}{\left.J_{0}\left(r k_{i}\right)\right|_{r_{\mathrm{ext}}}}-\left.\frac{1}{k_{i}} r \frac{\partial \widehat{h}_{2}}{\partial r}\right|_{r=1}\right), \quad \text { for ND-BCs, } \\
& =\mathcal{F}\left(k_{i}, s\right)\left(\left.r \frac{\partial \widehat{h}_{2}}{\partial r}\right|_{r_{\mathrm{ext}}} \frac{\left.J_{0}^{\prime}\left(r k_{i}\right)\right|_{r=1}}{\left.k_{i} J_{0}^{\prime}\left(r k_{i}\right)\right|_{r_{\mathrm{ext}}}}-\left.\frac{1}{k_{i}} r \frac{\partial \widehat{h}_{2}}{\partial r}\right|_{r=1}\right), \quad \text { for NN-BCs, }
\end{aligned}
$$

where $\tilde{x}$ is the finite Hankel transform of $x$ (Cinelli, 1965) and $\mathcal{F}\left(k_{i}, s\right)=2 /\left\{\pi\left[\eta(s)+k_{i}^{2}\right]\right\}$. After that, we take the inverse Laplace transform of Eq. (23), and then we use the inverse finite Hankel transform (Cinelli, 1965) to get

$$
\begin{array}{rlr}
h_{2}(r, t) & =\frac{\pi^{2}}{2} \sum_{k_{i}>0} \frac{k_{i}^{2} J_{0}^{2}\left(k_{i} r_{\mathrm{ext}}\right) \tilde{h}_{2}\left(k_{i}, t\right)}{J_{0}^{2}\left(k_{i}\right)-J_{0}^{2}\left(k_{i} r_{\mathrm{ext}}\right)} \mathcal{I}_{0,0}\left(k_{i}, r, 1\right), & \text { for DD-BCs, } \\
& =\frac{\pi^{2}}{2} \sum_{k_{i}>0} \frac{k_{i}^{2} J_{1}^{2}\left(k_{i} r_{\mathrm{ext}}\right) \tilde{h}_{2}\left(k_{i}, t\right)}{J_{0}^{2}\left(k_{i}\right)-J_{1}^{2}\left(k_{i} r_{\mathrm{ext}}\right)} \mathcal{I}_{0,0}\left(k_{i}, r, 1\right), \quad \text { for DN-BCs, } \\
& =\frac{\pi^{2}}{2} \sum_{k_{i}>0} \frac{k_{i}^{2} J_{0}^{2}\left(k_{i} r_{\mathrm{ext}}\right) \tilde{h}_{2}\left(k_{i}, t\right)}{J_{1}^{2}\left(k_{i}\right)-J_{0}^{2}\left(k_{i} r_{\mathrm{ext}}\right)} \mathcal{I}_{1,0}\left(k_{i}, 1, r\right), & \text { for ND-BCs, } \\
& =\frac{\pi^{2}}{2} \sum_{k_{i}>0} \frac{k_{i}^{2} J_{1}^{2}\left(k_{i} r_{\mathrm{ext}}\right) \tilde{h}_{2}\left(k_{i}, t\right)}{J_{1}^{2}\left(k_{i}\right)-J_{1}^{2}\left(k_{i} r_{\mathrm{ext}}\right)} \mathcal{I}_{1,0}\left(k_{i}, 1, r\right), & \text { for NN-BCs, }
\end{array}
$$


where $\mathcal{I}_{m, n}(x, y, z)=J_{m}(x y) Y_{n}(x z)-Y_{m}(x y) J_{n}(x z)$ and $k_{i}$ are the roots of $\mathcal{I}_{0,0}\left(k_{i}, 1, r_{\mathrm{ext}}\right), \mathcal{I}_{1,0}\left(k_{i}, r_{\mathrm{ext}}, 1\right)$, $\mathcal{I}_{1,0}\left(k_{i}, 1, r_{\text {ext }}\right)$, and $\mathcal{I}_{1,1}\left(k_{i}, 1, r_{\text {ext }}\right)$ for Eqs. (24a)-(24d), respectively. Equation (24) is for homogeneous inner BCs, since $\mathcal{I}_{0,0}\left(k_{i}, 1, r\right)=0$ and $\partial \mathcal{I}_{1,0}\left(k_{i}, 1, r\right) / \partial r=0$ when $r=1$. Therefore, it requires corrections when inhomogeneous BCs are considered. In Xi and Yuning (1991) a mathematical procedure is given to help with the correct application of the formulas of Cinelli (1965) when a hyperbolic differential equation is considered. However, in this work we choose to follow an alternative procedure, which only works when a long-time asymptotic (stationary) solution exists. Below, a discussion and evidences are presented. We propose the following procedure to correct Eq. (24):

- Regarding $\tilde{h}_{2}\left(k_{i}, t\right)$ in Eq. (24), this function is rewritten as follows:

$$
\tilde{h}_{2}\left(k_{i}, t\right)=\tilde{g}\left(k_{i}, t\right)+\tilde{\phi}\left(k_{i}\right)
$$

where $\tilde{\phi}\left(k_{i}\right)$ contains the time-independent (stationary) terms. Therefore, $\tilde{g}\left(k_{i}, t\right)$ is the transient part of the solution. For more details about $\tilde{g}\left(k_{i}, t\right)$ for each BCs case, see Appendix A.

- We write each equation in Eq. (24) as

$$
h_{2}(r, t)=\mathcal{H}^{-1}\left[\sum \tilde{g}\left(k_{i}, t\right)\right](r)+\mathcal{H}^{-1}\left[\sum \phi\left(k_{i}\right)\right](r),
$$

where $\mathcal{H}^{-1}\left[\sum \tilde{g}\left(k_{i}, t\right)\right](r)$ has the same form as the infinite sum in Eq. (24), considering that $\tilde{h}_{2}\left(k_{i}, t\right)$ is replaced by $\tilde{g}\left(k_{i}, t\right)$. This step is similar to the procedure in $\mathrm{Xi}$ and Yuning (1991), where the solution is divided in two parts.

- Since $\mathcal{H}^{-1}\left[\sum \phi\left(k_{i}\right)\right](r)$ is time independent, these terms are the stationary solution $h_{2, s}$ of the study model (3), i.e., the solution to the Laplace ordinary differential equation,

$$
\frac{1}{r} \frac{\partial}{\partial r}\left(r \frac{\partial h_{2, s}}{\partial r}\right)=0,
$$

with the inhomogeneous BCs in Table 1 at limit $t \rightarrow \infty$. Therefore,

$$
h_{2, s}(r)=\lim _{t \rightarrow \infty} h_{2}(r, t)=\mathcal{H}^{-1}\left[\sum \phi\left(k_{i}\right)\right](r)
$$

In this respect, it is worth noting that: 1) our procedure can be applied because the outer BC allows us to obtain a stationary solution for DD-BCs, DN-BCs, and ND-BCs cases; and 2) because in specific conditions there is no solution of Eq. (27) for the NN-BCs case, another alternative procedure to obtain the long-time solutions can be used. Namely, the long-time solution is obtained by expanding in series, about $s=0$, the equations in Table 2 and then taking the inverse Laplace transformation of these results, which lead us to the desired solution. We denoted this solution as $h_{2, q}=h_{2, q}(r, t)$, referring to a quasi-stationary solution.

- In view of the discussion above, the solution of model (3) is

$$
h_{2}(r, t)=h_{2, s}(r)+\mathcal{H}^{-1}\left[\sum \tilde{g}\left(k_{i}, t\right)\right](r),
$$

for DD-BCs, DN-BCs, and ND-BCs cases. Meanwhile, for the NN-BCs case, the solution is

$$
h_{2}(r, t)=h_{2, q}(r, t)+\mathcal{H}^{-1}\left[\sum \tilde{g}\left(k_{i}, t\right)\right](r) .
$$

Using the previous procedure (for additional details see Appendix A) the exact analytical solutions are

Volume 23, Issue 10, 2020 
DD-BCs case

$$
h_{2}(r, t)=1-\frac{\log (r)}{\log \left(r_{\mathrm{ext}}\right)}+\frac{\pi}{2} \sum_{i=1}^{\infty} \frac{\tilde{g}\left(k_{i}, t\right) \mathcal{I}_{0,0}\left(k_{i}, r, 1\right) J_{0}^{2}\left(r_{\mathrm{ext}} k_{i}\right)}{J_{0}^{2}\left(k_{i}\right)-J_{0}^{2}\left(r_{\mathrm{ext}} k_{i}\right)},
$$

and

$$
j_{2}(t)=\frac{1}{\log \left(r_{\mathrm{ext}}\right)}-\frac{\pi}{2} \sum_{i=1}^{\infty} \frac{k_{i} \tilde{g}\left(k_{i}, t\right) \mathcal{I}_{0,1}\left(k_{i}, 1,1\right) J_{0}^{2}\left(r_{\mathrm{ext}} k_{i}\right)}{J_{0}^{2}\left(k_{i}\right)-J_{0}^{2}\left(r_{\mathrm{ext}} k_{i}\right)} .
$$

DN-BCs case

$$
h_{2}(r, t)=1-q_{\mathrm{ext}} \log (r)+\frac{\pi^{2}}{2} \sum_{i=1}^{\infty} \frac{k_{i}^{2} \tilde{g}\left(k_{i}, t\right) \mathcal{I}_{0,0}\left(k_{i}, r, 1\right) J_{1}^{2}\left(r_{\mathrm{ext}} k_{i}\right)}{J_{0}^{2}\left(k_{i}\right)-J_{1}^{2}\left(r_{\mathrm{ext}} k_{i}\right)},
$$

and

$$
j_{2}(t)=q_{\mathrm{ext}}-\frac{\pi^{2}}{2} \sum_{i=1}^{\infty} \frac{k_{i}^{3} \tilde{g}\left(k_{i}, t\right) \mathcal{I}_{0,1}\left(k_{i}, 1,1\right) J_{1}^{2}\left(r_{\mathrm{ext}} k_{i}\right)}{J_{0}^{2}\left(k_{i}\right)-J_{1}^{2}\left(r_{\mathrm{ext}} k_{i}\right)} .
$$

ND-BCs case

$$
h_{2}(r, t)=\log \left(\frac{r_{\mathrm{ext}}}{r}\right)+\frac{\pi}{2} \sum_{i=1}^{\infty} \frac{\tilde{g}\left(k_{i}, t\right) \mathcal{I}_{1,0}\left(k_{i}, 1, r\right) J_{0}^{2}\left(r_{\mathrm{ext}} k_{i}\right)}{k_{i}\left[J_{1}^{2}\left(k_{i}\right)-J_{0}^{2}\left(r_{\mathrm{ext}} k_{i}\right)\right]}
$$

NN-BCs case

$$
\begin{aligned}
h_{2}(r, t) & =\frac{\pi^{2}}{2} \sum_{i=1}^{\infty} \frac{\tilde{g}\left(k_{i}, t\right) \mathcal{I}_{1,0}\left(k_{i}, 1, r\right) J_{1}^{2}\left(r_{\mathrm{ext}} k_{i}\right)}{J_{1}^{2}\left(k_{i}\right)-J_{1}^{2}\left(r_{\mathrm{ext}} k_{i}\right)}+\frac{2}{r_{\mathrm{ext}}^{2}-1}\left(\frac{r^{2}}{4}+t\right) \\
& -\frac{r_{\mathrm{ext}}^{2}}{r_{\mathrm{ext}}^{2}-1} \log (r)-\frac{3 r_{\mathrm{ext}}^{4}-4 r_{\mathrm{ext}}^{4} \log \left(r_{\mathrm{ext}}\right)-2 r_{\mathrm{ext}}^{2}-1}{4\left(r_{\mathrm{ext}}^{2}-1\right)^{2}}+q_{\mathrm{ext}}\left[\frac{2}{r_{\mathrm{ext}}^{2}-1}\left(\frac{r^{2}}{4}+t\right)-\frac{\log (r)}{r_{\mathrm{ext}}^{2}-1}\right. \\
& \left.-\frac{r_{\mathrm{ext}}^{4}+2 r_{\mathrm{ext}}^{2}-4 r_{\mathrm{ext}}^{2} \log \left(r_{\mathrm{ext}}\right)-3}{4\left(r_{\mathrm{ext}}^{2}-1\right)^{2}}\right]+\frac{2 \gamma q_{\mathrm{ext}}\left(1-\mathrm{e}^{-t / \gamma}\right)}{r_{\mathrm{ext}}^{2}-1} .
\end{aligned}
$$

\section{ANALYSIS OF THE SOLUTIONS}

The analytic solutions of Eq. (3) are numerically validated. With this aim, numerical results are obtained by means of the Stehfest (1970) method, which is used to take the inverse Laplace transform of equations in Table 2. These results are compared with the results from the following relationships: 1) Eq. (24) or Cinelli solutions and 2) Eqs. (31)-(36) or Cinelli solutions with closed relationships at large time. An analysis of the existence of long-term solutions is also presented.

\subsection{Validation of the Analytic Solutions Using Stehfest Method}

Figure 2 shows the matching between the results from analytic solutions, Eqs. (32), (34), (35), and (36), and the results from the inversion of Eqs. (38), (40), (41), and (43), respectively. Values of the parameters are given inside the frames of the figure. Figures 2(a) and 2(b) have graphs of flux into the wellbore, while Figs. 2(c) and 2(d) have graphs of drawdown pressure at the bottomhole. They exhibit the different flow regimes, which for Figs. 2(a) and 2(b) are related to fractures, transition fractures-matrix, and matrix, and they are presented at early, middle, and long times, respectively (Warren and Root, 1963). On the other hand, Figs. 2(b) and 2(d), in addition, involve a stage dominated by recharge boundary effects, which are presented for a long-time production (del Angel et al., 2014; Doublet and Blasingame, 1995; Wang et al., 2017). Therefore, these latter figures include graphs with a transition stage between homogeneous behavior and influx recharge. Note that the transition stages between media with different permeabilities occur around the inflection points (Uldrich and Ershaghi, 1979). In Fig. 2(b), we note that the stage dominated by the transition fracture-matrix is dimmed because the influx recharge effects arise close to this transition 
TABLE 2: Exact solutions in Laplace space of the fluid flow equation [Eq. (3)] and their different BCs cases

\begin{tabular}{|c|c|c|c|c|}
\hline Case & Pressure $^{\dagger}$ & & Flux $^{\ddagger}$ & \\
\hline DD-BC & $\widehat{h}_{2}(r, s)=\frac{\Psi_{0,0}\left(r_{\mathrm{ext}}, r, \sqrt{\eta(s)}\right)}{s \Psi_{0,0}\left(r_{\mathrm{ext}}, 1, \sqrt{\eta(s)}\right)}$ & (37) & $\widehat{j}_{2}(s)=\frac{\sqrt{\eta(s)} \Psi_{0,1}\left(r_{\mathrm{ext}}, 1, \sqrt{\eta(s)}\right)}{s \Psi_{0,0}\left(1, r_{\mathrm{ext}}, \sqrt{\eta(s)}\right)}$ & (38) \\
\hline DN-BC & $\begin{array}{r}\widehat{h}_{2}(r, s)=\frac{\Psi_{1,0}\left(r_{\mathrm{ext}}, r, \sqrt{\eta(s)}\right)}{s \Psi_{0,1}\left(1, r_{\mathrm{ext}}, \sqrt{\eta(s)}\right)} \\
+\frac{\widehat{f}(s)}{r_{\mathrm{ext}}} \frac{\Psi_{0,0}(1, r, \sqrt{\eta(s)})}{\sqrt{\eta(s)} \Psi_{0,1}\left(1, r_{\mathrm{ext}}, \sqrt{\eta(s)}\right)}\end{array}$ & (39) & $\begin{array}{r}\widehat{j}_{2}(s)=\frac{\sqrt{\eta(s)} \Psi_{1,1}\left(1, r_{\mathrm{ext}}, \sqrt{\eta(s)}\right.}{s \Psi_{0,1}\left(1, r_{\mathrm{ext}}, \sqrt{\eta(s)}\right)} \\
-\frac{\widehat{f}(s)}{r_{\mathrm{ext}}} \frac{\Psi_{0,1}(1,1, \sqrt{\eta(s)})}{\Psi_{0,1}\left(1, r_{\mathrm{ext}}, \sqrt{\eta(s)}\right)}\end{array}$ & (40) \\
\hline ND-BC & $\widehat{h}_{2}(r, s)=\frac{\Psi_{0,0}\left(r, r_{\mathrm{ext}}, \sqrt{\eta(s)}\right)}{s \sqrt{\eta(s)} \Psi_{0,1}\left(r_{\mathrm{ext}}, 1, \sqrt{\eta(s)}\right.}$ & (41) & $\widehat{j}_{2}(s)=\frac{1}{s}$ & $(42)$ \\
\hline NN-BC & $\begin{array}{r}\widehat{h}_{2}(r, s)=\frac{\Psi_{0,1}\left(r, r_{\mathrm{ext}}, \sqrt{\eta}(s)\right)}{s \sqrt{\eta(s)} \Psi_{1,1}\left(1, r_{\mathrm{ext}}, \sqrt{\eta(s)}\right.} \\
+\frac{\widehat{f}(s)}{r_{\mathrm{ext}}} \frac{\Psi_{0,1}(r, 1, \sqrt{\eta(s)})}{\sqrt{\eta(s)} \Psi_{1,1}\left(1, r_{\mathrm{ext}}, \sqrt{\eta(s)}\right)}\end{array}$ & (43) & $\widehat{j}_{2}(s)=\frac{1}{s}$ & (44) \\
\hline
\end{tabular}

${ }^{\dagger}$ Problem statement is given in Eq. (21) with the BCs (15)-(19).

${ }^{\ddagger} \Psi_{m, n}(\Phi, \Upsilon, x)=K_{m}(\Phi x) I_{n}(\Upsilon x)+(-1)^{m+n+1} I_{m}(\Phi x) K_{n}(\Upsilon x)$ and $\eta(s)=\{[s \omega(1-\omega)+\lambda] s\} /[s(1-\omega)+\lambda]$.
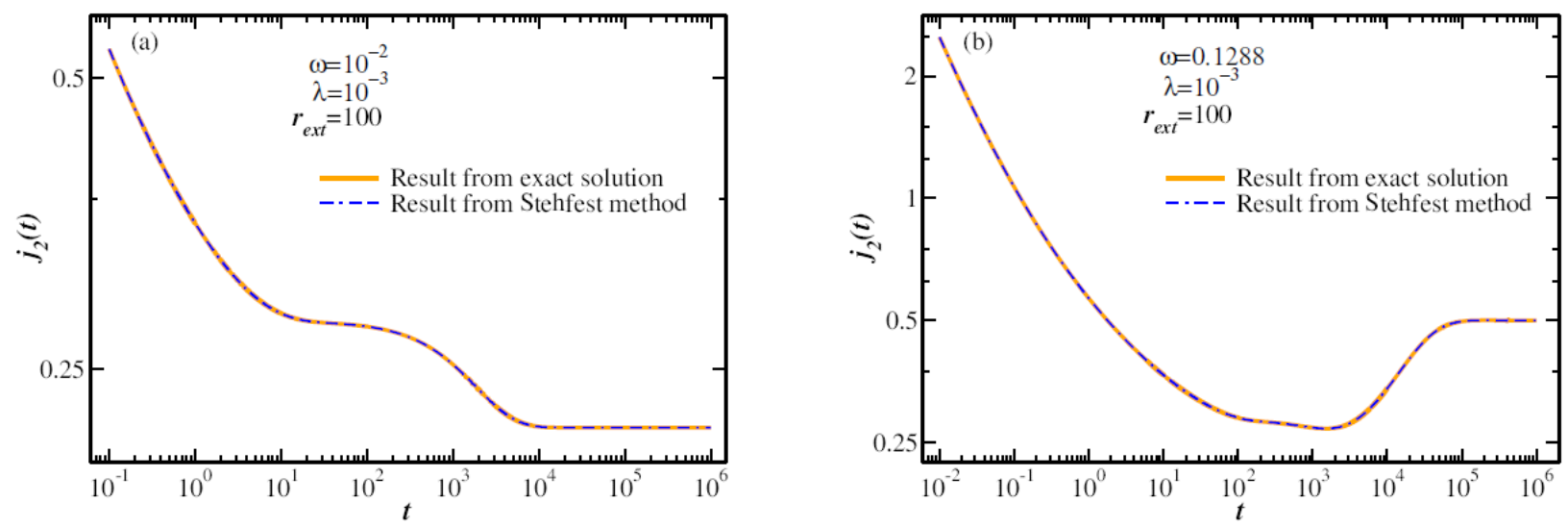

FIG. 2. 

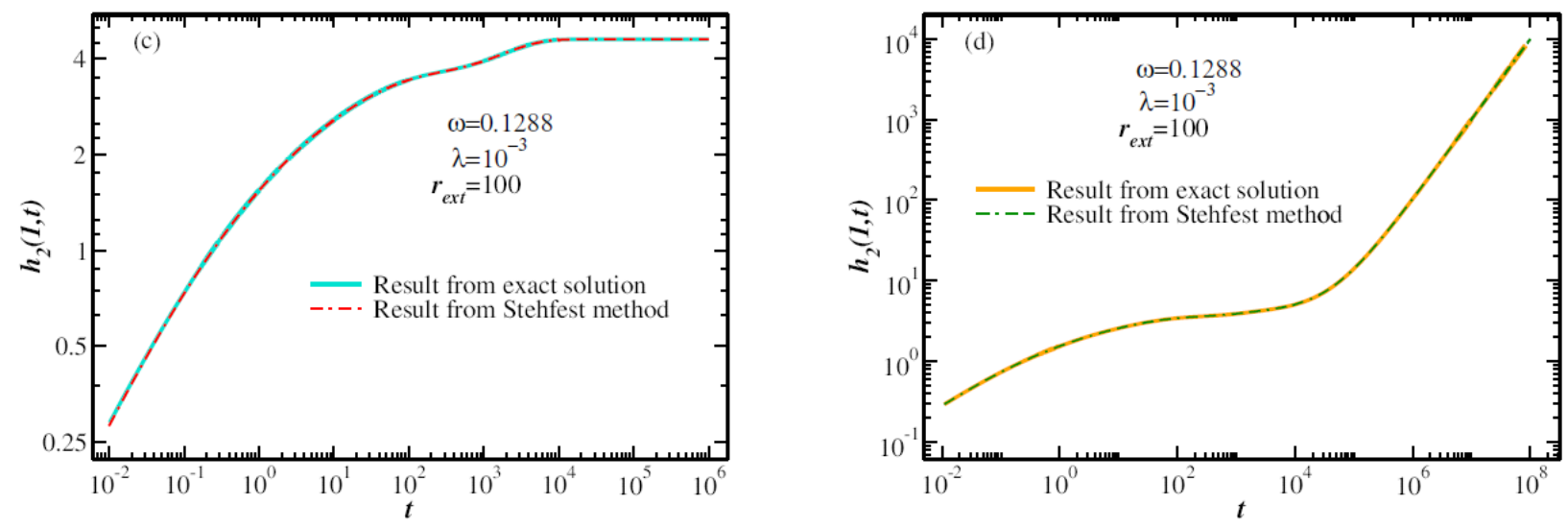

FIG. 2: A comparison between the exact solutions from JLHT and numerical solutions from the Stehfest method is shown. A matching is observed between both results. Equations (32), (34), (35), and (36) are used to obtain the results in the subfigures for the cases (a) DD-BCs, (b) DN-BCs, (c) ND-BCs, and (d) NN-BCs, respectively, while the results from the inverse Laplace transform are obtained by making use of Eqs. (38), (40), (41), and (43). The parameter values used for the ramp rate function are $q_{\mathrm{ext}}=0.5$ and $\gamma=10^{-3}$. In (a), (b), (c), and (d) 100, 100, 1000, and 200 are used $k_{i}$-roots, respectively.

stage. In summary, the expected physical behavior of a slightly compressible fluid in a double-porosity medium is observed, as well as a perfect matching, to the naked eye, between the numerical and theoretical results. It is worth mentioning that a similar conclusion is obtained for other parameter choices.

\subsection{Convergence of the Cinelli and Analytical Solutions}

In addition to validating the analytical solutions, they are reduced to classical solutions reported in the literature: Muskat (1934), for DD-BCs; Hurst (1934) and Muskat (1934), for DN-BCs; Matthews and Russell (1967), for ND-BCs; and del Angel et al. (2014); Matthews and Russell (1967); Muskat (1934), for NN-BCs. Based on this observation and the numerical validation of the previous section, we assume that Eqs. (31)-(36) are correct. For the convergence analysis, we consider that the results of Eq. (31) are exact when the truncation involves $10^{4}$ terms, rounding results to six decimal place accuracy (this precision is established in such a way that the floating numerical result would not change when new terms are added to the solution in infinite series). The error behavior in function of the number of terms can be seen in Fig. 3(a), which exhibits errors with less than five orders of magnitude. Similar results should be found for the other study cases (not shown in the figure) as well as for other $t$ values. Therefore, for practical purposes, the results in Fig. 3(a) suggest that a truncation with 100 terms can be used to get a good accuracy. In general, for Eqs. (31)-(36), this number of terms is suitable for obtaining reliable results for applications. Likewise, the error associated with the Stehfest method is computed, as can be seen in Fig. 3(b). This latter error has amplitudes and oscillations greater than the error from Eq. (31), and in fact, it is important to mention that there is no control over the error obtained with the Stehfest method.

A related point to consider is the convergence analysis of Eq. (24), whose behaviors can be seen in Fig. 4. In this figure it is remarkable the systematic convergence of the results by truncating the series in Eq. (24) with a greater number of terms. Also, notice that the results tend to zero at the inner boundary and that their convergence is very slow close to this boundary. In addition, note that the oscillations are increased by increasing the number of terms, as can be seen in Figs. 4(a) and 4(b). There are techniques to speed up the convergence rate (Shanks, 1955), but we show that the solutions with the closed formulas also speed up the convergence, as indicated by the running times given in Table 3 (the computational cost to make an inversion with the Stehfest method is also included). Figures 4(a) and 4(b) contain graphs of the DD-BCs and DN-BCs cases when $10^{3}, 10^{4}$, and $10^{5}$ terms or roots are considered in the computations. Despite the great increase in the number of roots, the results have considerable oscillations around the numerical results. Note that these latter numerical results match with the ones from Eqs. (31) and (33) and are 

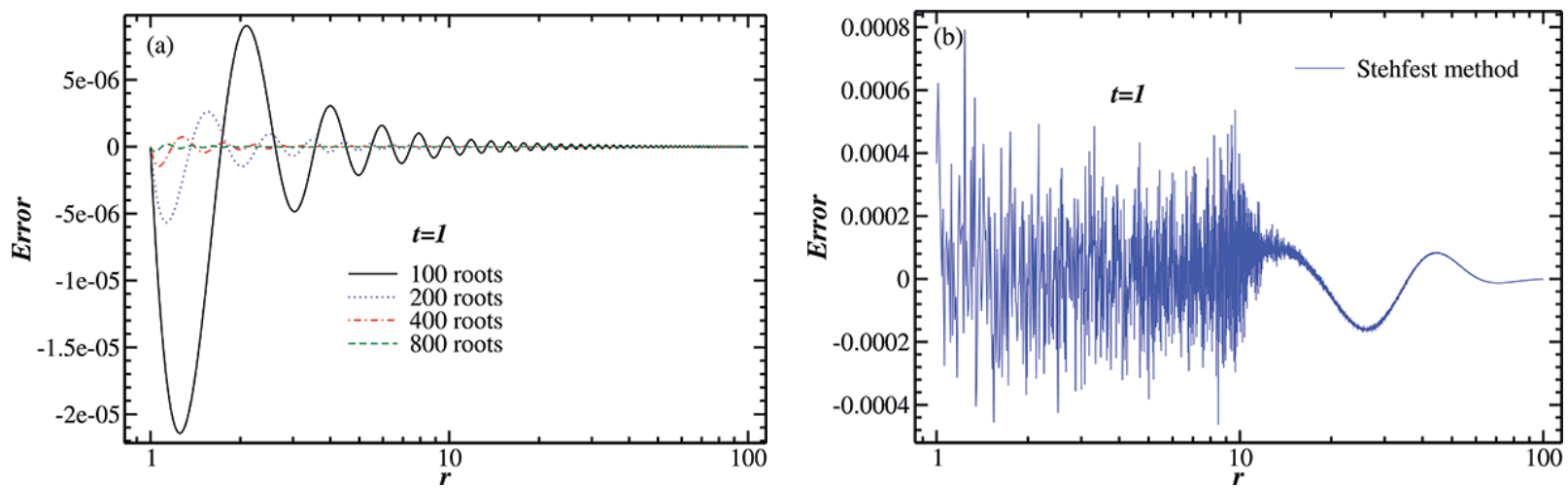

FIG. 3: Numerical error of DD-BCs case. The results from Eq. (31) truncated with $10^{4}$ terms are subtracted from (a) the results of Eq. (31) truncated with the number of terms as indicated in the frame and (b) the results of the inversion of Eq. (37). In addition, the following parameter values are used: $\omega=10^{-2}, \lambda=10^{-3}$, and $r_{\text {ext }}=100$.
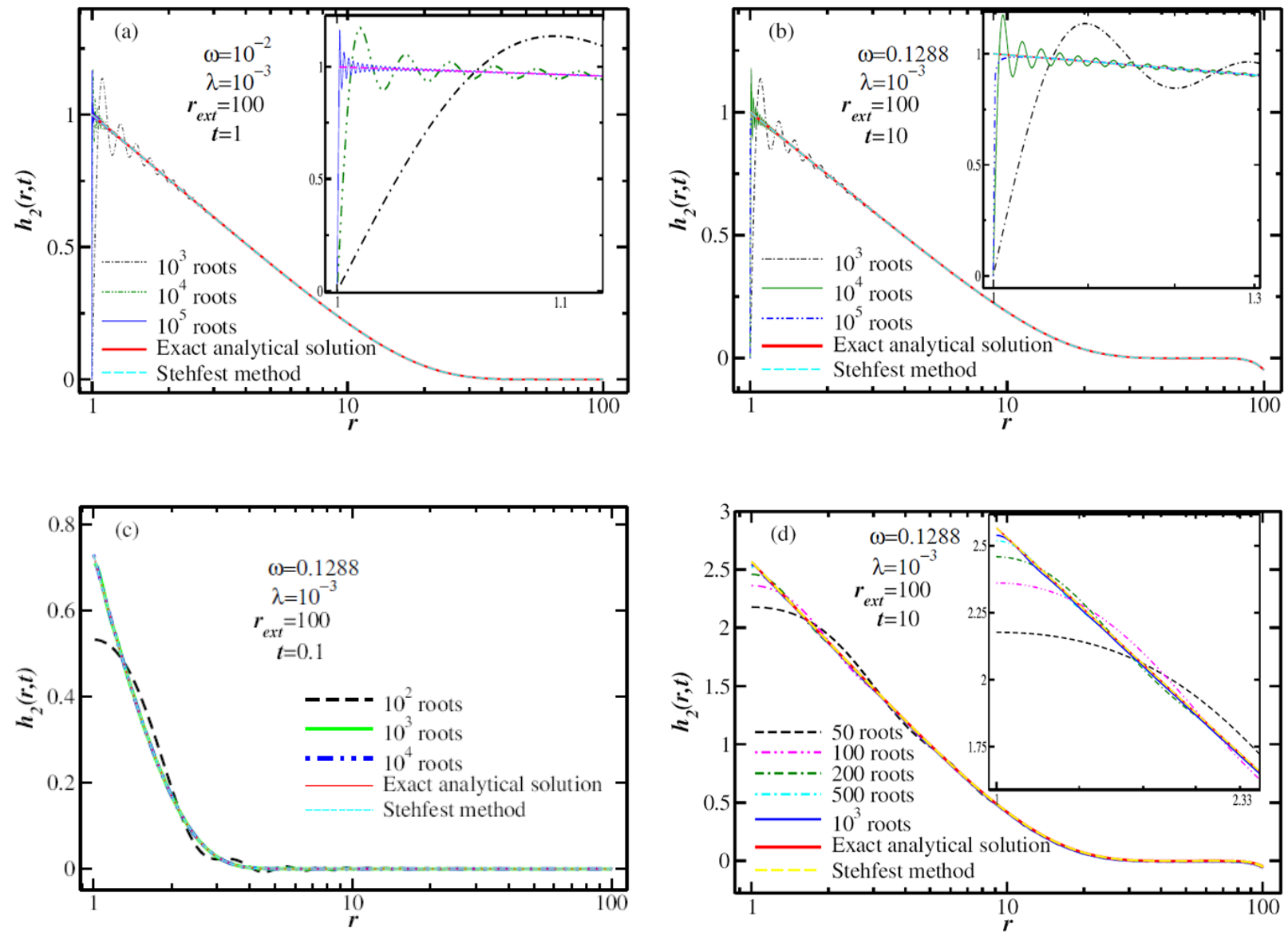

FIG. 4. 

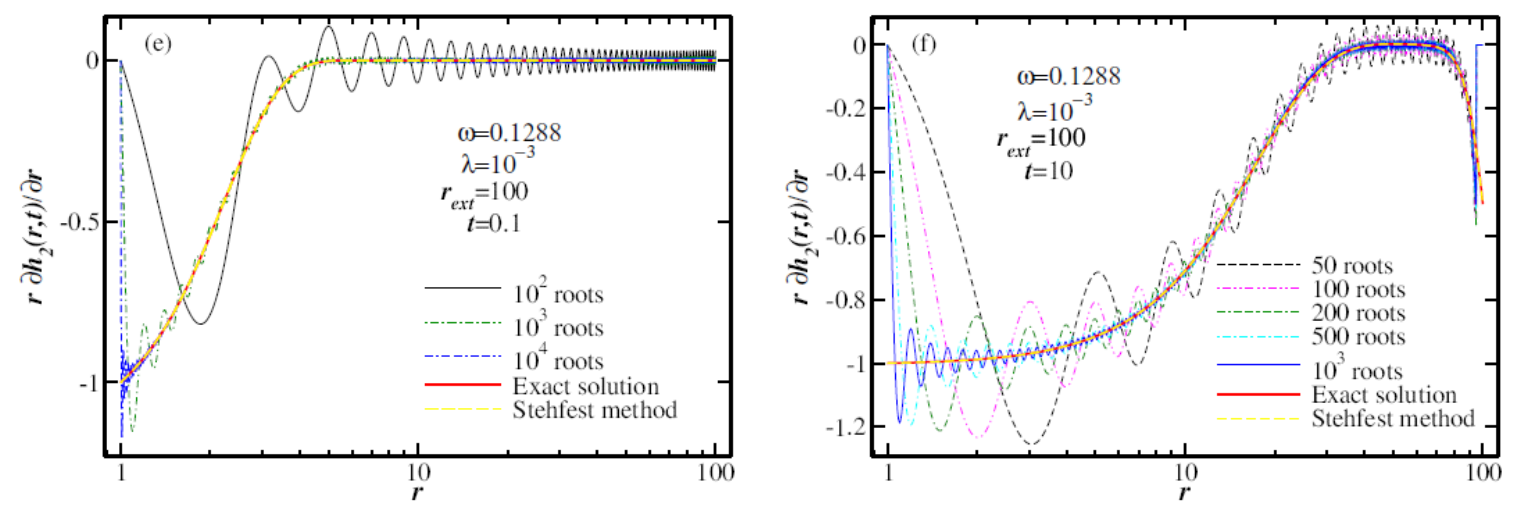

FIG. 4: Results of the exact pressure (our $h_{2}(r, t)$ formulas) for the cases: (a) DD-BCs, (b) DN-BCs, (c) ND-BCs, and (d) NNBCs, and exact flux $j_{2}(r, t)$ for the cases (e) ND-BCs, and (f) NN-BCs. These results are compared with the corresponding ones obtained with Eqs. (24) and with the Stehfest method applied to the equations of Table 2. It is remarkable how the results from Eqs. (24), except for subfigures (c) and (d), show a slow converge and oscillations around the exact results, and also that the inner boundary condition is not hold in any case. For (c) and (d), the oscillatory behavior is diminished and the convergence is faster than in the other subfigures; note that in these cases the inner boundaries have flux conditions which never are meet. In all cases is observed a perfect matching between the exact solutions and the Stehfest method results.

TABLE 3: Computational $\cos ^{\S}$ (in seconds) to obtain a results of $h_{2}(r, t)$

\begin{tabular}{c|c|c|c}
\hline Case & Cinelli $\left(\mathbf{1 0}^{\mathbf{4}}\right.$ roots $)$ & This work $\left(\mathbf{1 0}^{\mathbf{2}}\right.$ roots $)$ & Stehfest \\
\hline DD-BCs & 1.40 & 0.0001 & $4.8 \times 10^{-5}$ \\
DN-BCs & 0.90 & 0.0086 & $8.8 \times 10^{-5}$ \\
ND-BCs & 0.01 & 0.0001 & $5.2 \times 10^{-5}$ \\
NN-BCs & 0.04 & 0.0003 & $4.4 \times 10^{-5}$ \\
\hline
\end{tabular}

${ }^{\S}$ Running time scales linearly with the number of terms used to compute the result.

free of oscillations. On the other hand, Figs. 4(c) and 4(d) contain graphs for ND-BCs and NN-BCs cases. Again, we observed a systematic convergence by increasing the number of terms. In fact, for ND-BCs and NN-BCs cases, the oscillation quickly diminishes when more terms of the series are considered in the computation. Even so, the number of terms is large compared to the number of terms used in the computation by means of equations that involve the closed formulas, Eqs. (35) and (36). For example, in Fig. 2 hundreds of terms are used, while in Fig. 4 thousands. However, the behavior like the one given for Figs. 4(a) and 4(b) is found when flux along the reservoir (at fixed time) is plotted, see Figs. 4(e) and 4(f). This is because these graphs show the convergence of the solution toward the value of the inner BC. It is worth remarking that results in Fig. 4 are computed either with the solutions of Cinelli or the exact ones, and that both have infinite sums. Therefore, the absence of oscillations in the exact solutions is due to the fact that the infinite sums (corresponding to the stationary limit) could be approximated with simplified analytical expressions, i.e., the oscillations are attributable to computations with a finite number of terms of the timeindependent series. In summary, it is remarkable that: 1) direct use of Eq. (24) leads to the correct results, except at the inner boundary, and 2) when the closed formulas are used, the inhomogeneous BCs hold independently of the number of terms.

\subsection{Existence of Stationary Solutions}

As we have highlighted, the solution of

$$
\frac{1}{r} \frac{\partial}{\partial r}\left(r \frac{\partial h_{2, s}}{\partial r}\right)=0, \quad \text { which is } \quad h_{2, s}(r)=A \log (r)+B,
$$


is involved in Eq. (29), and this is stationary. For this reason, the solution of model (3) is represented as the sum of a stationary solution and a transitory solution. This latter solution has the same form as the Cinelli formulas, replacing $\tilde{h}_{2}\left(k_{i}, t\right)$ in Eq. (24) by $\tilde{g}\left(k_{i}, t\right)$. Therefore, the transitory part holds by default for homogeneous BC, and, for this reason, the stationary part $h_{2, s}(r)$ should reproduce the inner BC.

Because of the nonuniqueness condition required for a well-posed boundary value problem, Eq. (45) has no stationary solution when a fixed flow is imposed at the inner boundary with an influx through the outer boundary. Solution methods can lead to uniqueness by specifying a conservation principle or splitting the problem into wellposed problems [see, for example, exercises 18.3.10 (c) and 18.3.19 in Greenberg (1998)]. Regarding this remark, stationary solutions arise when the inner and outer boundary conditions are equal, i.e., when $A$ has the same value in both boundaries. Thus, the flux has a long-time stationary behavior because no net flow enters or leaves the reservoir. Under these conditions, we have validated that our methodology, in relation with Eq. (29), works well. Otherwise, when the net flux in the reservoir increases or decreases at any time, a dynamic behavior predominates over time. In this case, we have observed that the formula in Cinelli (1965) for NN-BCs is limited to work well when no net flux leaves or enters the reservoir, while inconsistencies are observed when the influx recharge has a predominant effect in the system at large times. In fact, the flattened line in Fig. 5 exhibits such inconsistency, since the results given by this formula remain constant for long time, for any value of $q_{\text {ext }}$ chosen. This behavior disagrees with the one obtained using the Stehfest method, in which there is an increase in the asymptotic behaviors when $q_{\text {ext }}<1$ and a decrease when $q_{\text {ext }}>1$. These characteristics are consistent with those observed in van Everdingen and Hurst (1949) and del Angel et al. (2014) and, as we can see in Fig. 5, they are reproduced by our exact solution, Eq. (36), which is valid for any value of $q_{\text {ext }}$ considered. Notice that some flow problems with NN-BCs have been successfully solved with the Weber transform (Babak and Azaiez, 2014; Tong and Hu, 2010; Zhang and Tong, 2007).

It is worth mentioning that we have deliberately added the time-dependent terms on the right-hand side of Eq. (36) to obtain the correct behavior; these terms are identified by being outside of the infinite series indicated there. As it was stated in Section 4, these added terms are obtained by expanding Eq. (43) about $s=0$, and, subsequently, taking the inverse Laplace transform to the result of this expansion. It can be seen that these terms are not considered in the Cinelli relationships, Eq. (24d), and their inclusion leads to a correct matching with the numerical results, as can be seen in Fig. 5. Similarly, it can be proved that stationary solutions for DD-BCs, DN-BCs, and ND-BCs cases can be recovered using this procedure.

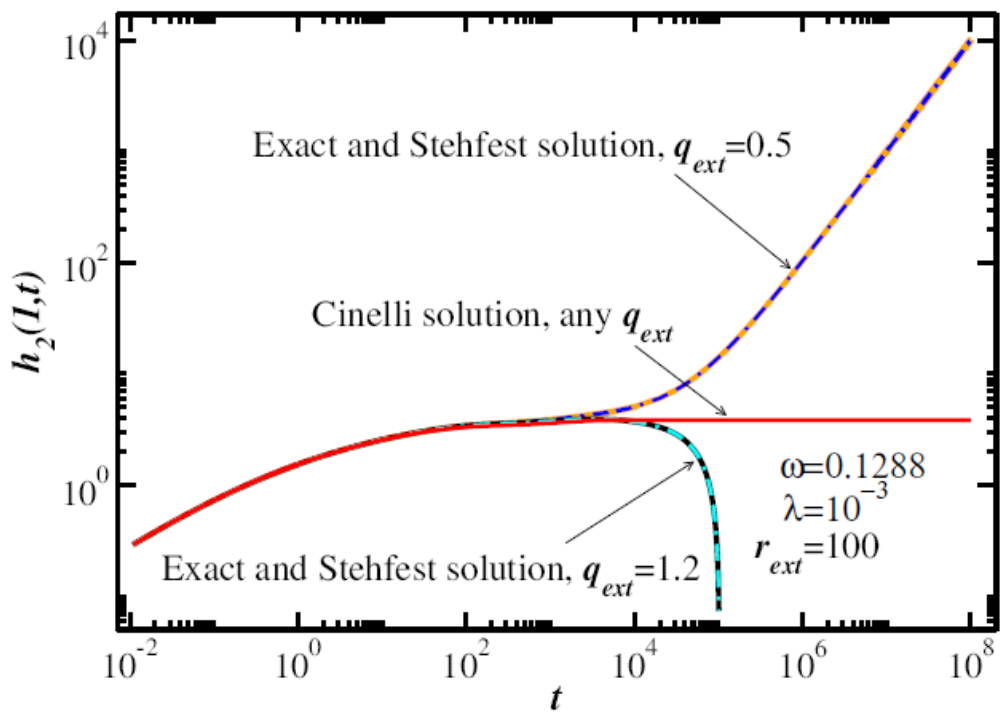

FIG. 5: Drawdown pressure curves for the NN-BCs case. The flattened line is the result from the Cinelli formula [Eq. (24d)], and the dashed lines are obtained using the exact result given by Eq. (36). For comparison, the inverted numerical results from Eq. (43) are also shown in dashed lines. In all cases $3 \times 10^{4}$ terms are used. The considered $\gamma$ value is $10^{-3}$. 


\section{CHARACTERISTIC BEHAVIOR OF RESERVOIRS WITH INFLUX RECHARGE}

In this section, characteristic curves of drawdown pressure and flux for reservoirs with influx recharge are presented. Drawdown pressure curves are analyzed in the framework of the pressure derivative of Bourdet (Bourdet et al., 1983, 1989). Subsequently, a comparative analysis between models with NN-BCs is done, considering a model of a singleporosity and nonzero influx and a second model of double-porosity but with a closed boundary. This analysis is done with the purpose of elucidating whether they can be quantitatively equivalent.

It is worth mentioning, before beginning the analysis, that for naturally fractured reservoirs, usual values of the interporosity flow coefficient are $10^{-10} \leq \lambda \leq 10^{-4}$, while the fracture storage coefficient $\omega$ is in the order of $10^{-3}$ or $10^{-2}$ Bourdet (2002). Regarding the work of Kuhlman et al. (2015), we have $\omega \lesssim 0.1 \%$. The parameter $q_{\text {ext }}$ (or $\gamma)$ has not been characterized because of the scarcity of studies in this direction. On the other hand, according to the Warren and Root model, the parameter values admit the following ranges: $0<\omega \leq 1, \lambda>0, q_{\mathrm{ext}} \geq 0$, and $\gamma>0$. In a similar way to other works (Mavor and Cinco-Ley, 1979; Wang et al., 2017), we use these latter ranges to show the characteristic behavior of the study model. Indeed, best-fitting curves of the Warren and Root model could lead to a large value of $\omega$, e.g., Camacho Velazquez et al. (2014).

\subsection{Characteristic Curves}

\subsubsection{DN-BCs Case}

Taking into account that the condition imposed at the bottomhole is constant pressure, Eq. (34) leads to the flux behaviors shown in Fig. 6. Therein, the differences between the graphs come from varying the values of the storage $\omega$, the interporosity flux coefficient $\lambda$, the slope of the ramp $\gamma$, the outer influx factor $q_{\text {ext }}$, and the outer radius $r_{\text {ext }}$. Note that the interporosity flow coefficient is a first-order mass transfer coefficient Goltz and Oxley (1991); Moench (1995). In Fig. 6(a) it is evident that at early times, $\omega$ has a major effect on the flux. Namely, a small $\omega$ leads to a transition fracture-matrix more pronounced, while the graph for $\omega=0.9$ does not show the characteristic form of such transition. Figure 6(a) also exhibits that, for fixed $\omega$, this form is affected by changing the $\lambda$ value. The $\lambda$ effect in the flux can also be seen in Fig. 6(b); for $\lambda \geq 10^{-1}$, transitions with a negative half-slope are observed, while the graphs for the rest of the values of $\lambda$ are similar to the ones from a single-porosity medium. On the other hand, since $\gamma$ is an influx parameter, its effect is seen in Fig. 6(c) for a long time production. Because a large $\gamma$ implies a slow influx recharge into the reservoir, the flux declination is more pronounced when the $\gamma$ value is increased. In addition, the effect of $r_{\text {ext }}$ becomes clear in Fig. 6(d). Namely, the characteristic curves show that a larger $r_{\text {ext }}$ leads to a greater flux drop. In this figure, we give three graphs for each value of $r_{\text {ext }}$, each of these depending on a couple of values of $q_{\text {ext }}$ and $\omega$ as indicated there. Notice that the flux drop is recovered at long time when the flux becomes stationary with a $q_{\text {ext }}$ value; it can be seen for every graph in Fig. 6 . We do not include solutions for a closed reservoir, $q_{\text {ext }}=0$ : however, for this case the flux tends to zero, and the solutions have no minimums as show in Fig. 6.

\subsubsection{NN-BCs Case}

Examples of drawdown pressures and their Bourdet derivatives are found in Fig. 7. We can see how changes in $\omega$ have effect for early time; the effect by varying $\lambda$ covers the solution domain, while changes due to $\gamma$ are presented for long time; see Figs. 7(a)-7(c), respectively. Similar characteristic behaviors have been observed in other works (del Angel et al., 2014; Wang et al., 2017); however, these works were not for double-porosity media. The drawdown pressure shows several stages as time evolves, which are influenced by fractures, transition fractures-matrix, matrix, transition matrix-influx, and influx. Furthermore, the Bourdet derivatives have minimums in correspondence with the transition stages. It can be noticed in Fig. 7 that both characteristic curves have the same linear behavior at long times when log-log graphs are considered, with a slope value of 1 . However, note that this fact leads to an extension of the range of the matrix stage when $\lambda$ is very small, as can be seen for $\lambda=10^{-7}$ in Fig. 7(b). A similar situation occurs in Fig. 7(c), where $\gamma=10^{5}$ implies a longer time for the recharge to take effect. In addition, the effect of $r_{\text {ext }}$ becomes clear in Fig. 7(d). Namely, the characteristic curves show that for a high $r_{\text {ext }}$ and $t$, the drawdown behaviors are that of the Warren and Root model. In this figure, we give a set of graphs for each value of $r_{\text {ext }}$ and different values of $q_{\text {ext }}$; 

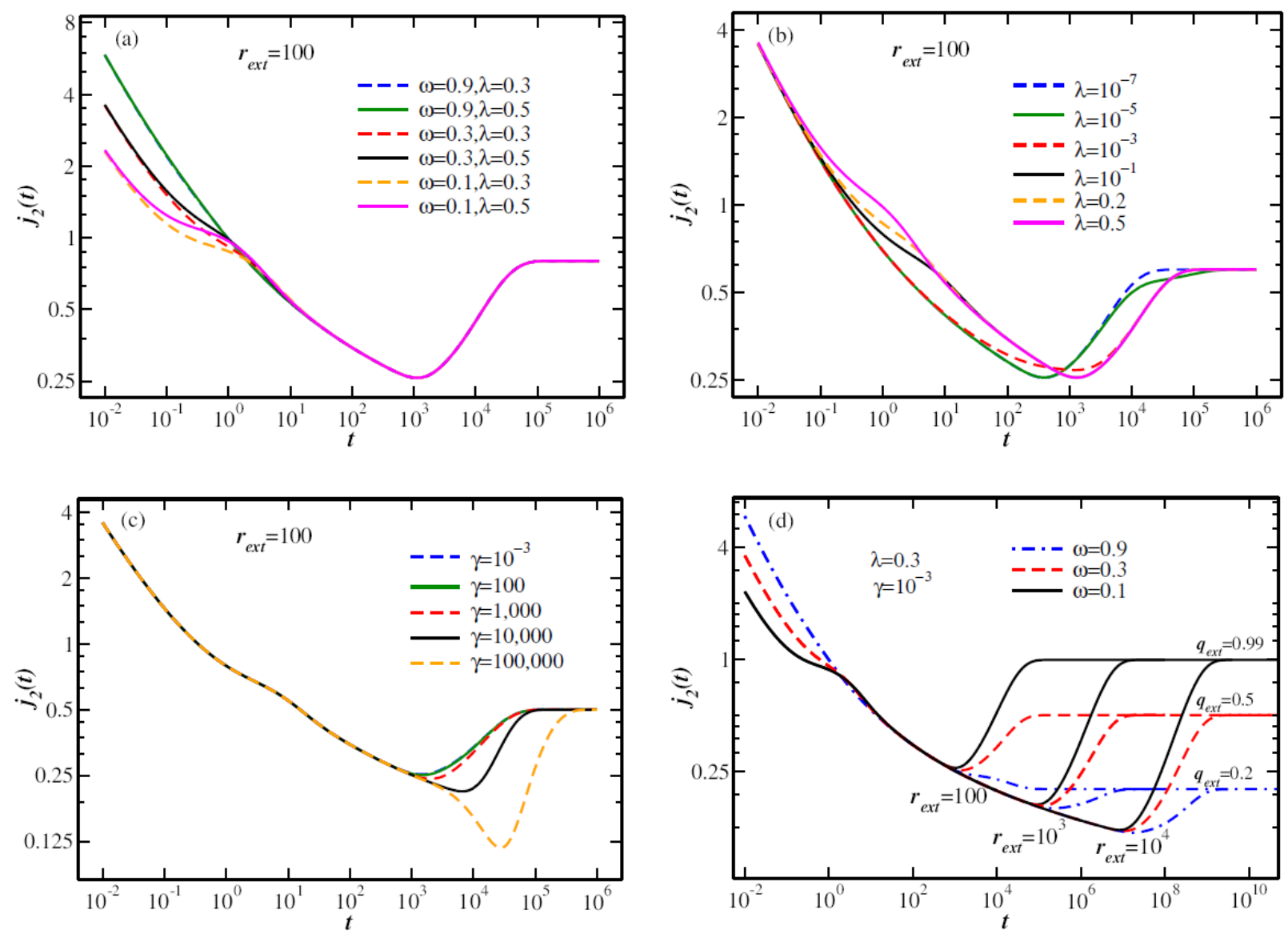

FIG. 6: Flux characteristic curves obtained from Eq. (34) for the DN-BCs case. The frames show the effect of the following parameters on the curves: (a) $\omega$ and $\lambda$, (b) $\lambda$, (c) $\gamma$, and (d) $\omega, r_{\text {ext }}$, and $q_{\text {ext }}$. The rest of the parameters values, in addition to the ones given in frames, are: (a) $q_{\mathrm{ext}}=0.8$ and $\gamma=10^{-3}$, (b) $\omega=0.3, q_{\mathrm{ext}}=0.6$ and $\gamma=10^{-3}$, (c) $\omega=0.3, q_{\mathrm{ext}}=0.5$ and $\lambda=0.1$.
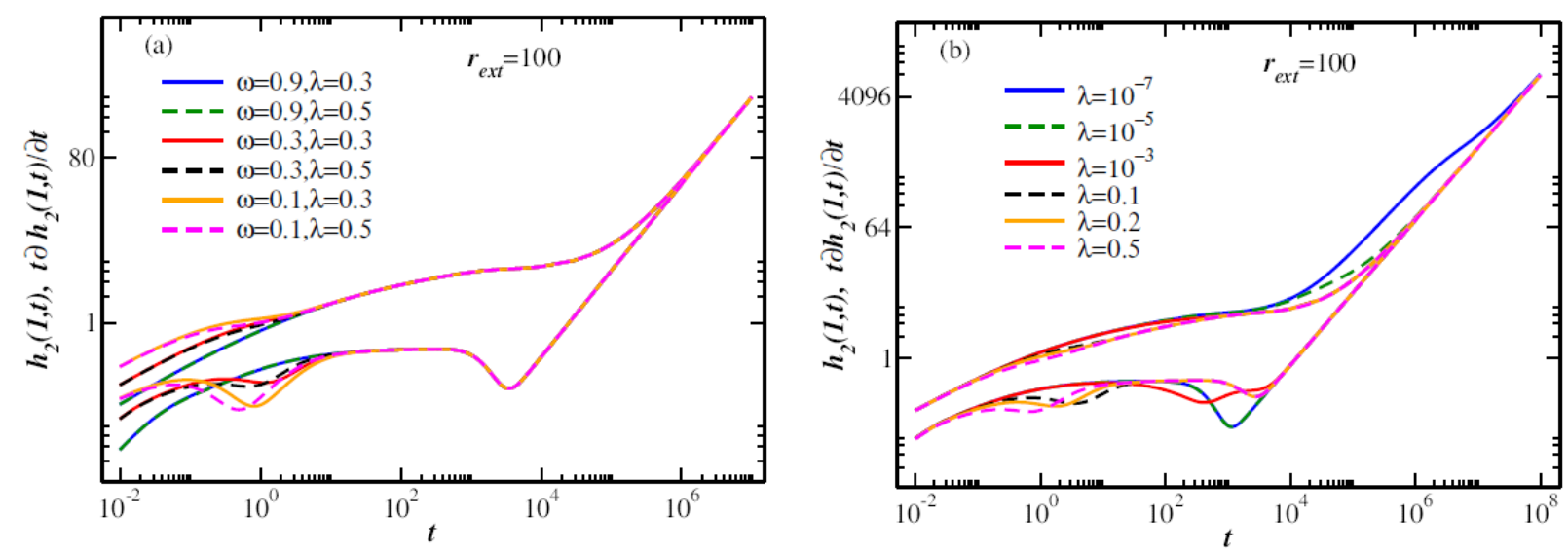

FIG. 7. 

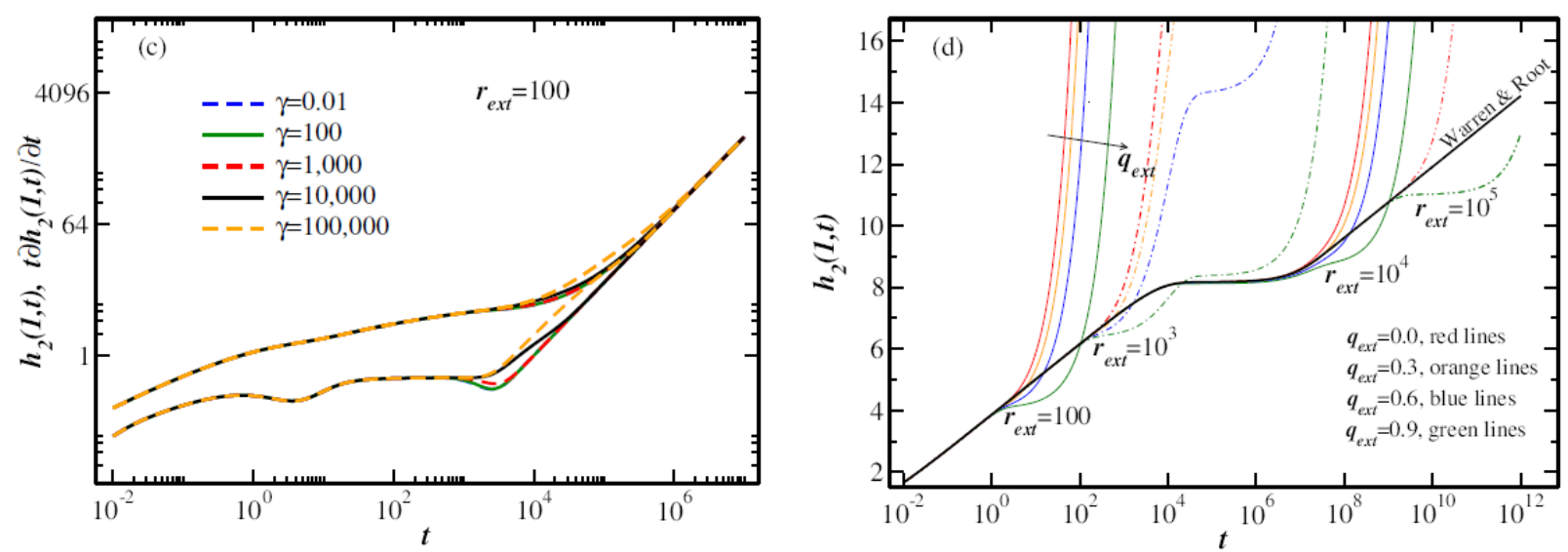

FIG. 7: Drawdown pressure characteristic curves, Eq. (36), and their Bourdet derivatives for the NN-BCs case. The frames show the effect of the following parameters on the curves: (a) $\omega$ and $\lambda$, (b) $\lambda$, (c) $\gamma$, and (d) $r_{\text {ext }}$ and $q_{\text {ext }}$. The rest of the parameter values, in addition to the ones given in frames, are: (a) $q_{\text {ext }}=0.8$ and $\gamma=10^{-3}$, (b) $\omega=0.3, q_{\text {ext }}=0.6$, and $\gamma=10^{-3}$, (c) $\omega=0.3$, $q_{\mathrm{ext}}=0.5$, and $\lambda=0.1$, and (d) $\omega=0.001, \lambda=10^{-7}$, and $\gamma=10^{-3}$.

each of these sets is indicated by different types of line. Notice also that the drawdown behavior is that of a closed reservoir, except when $r_{\mathrm{ext}} \rightarrow \infty$, i.e., the behavior at long time of a closed reservoir, or $q_{\mathrm{ext}}=0$, is similar to that of a reservoir with influx recharge when $0<q_{\text {ext }}<1$.

As mentioned above, the drawdown pressure and its Bourdet derivative have the same asymptotic curve at long times. Indeed, this is obtained from Eq. (36) and its derivative,

$$
t \frac{\partial h_{2}}{\partial t}=\left(\frac{2}{r_{\mathrm{ext}}^{2}}-\frac{2 q_{\mathrm{ext}}}{r_{\mathrm{ext}}^{2}-1}\right) t=h_{2} .
$$

In the previous result, we use the fact that the transitory part of the solution tends to zero when time increases, as we deduced before in the discussion of Fig. 5. The influence of the parameter $q_{\text {ext }}$ on pressure drawdown curves is analyzed in del Angel et al. (2014) and Wang et al. (2017), where a single-porosity and triple-porosity reservoir are studied, respectively. In these works it is observed that the influx recharge has no influence on the early and middle production stages. Furthermore, the following points are remarkable: 1 ) when $q_{\mathrm{ext}}=1$ it implies $\partial h_{2} / \partial t=0$, i.e., there is a stationary solution with a constant pressure at the outer boundary; 2) when $q_{\mathrm{ext}}<1$ there is a pseudo-steadystate solution, and the influence of $q_{\mathrm{ext}}$ is similar to the case when there is a closed boundary; and 3) when $q_{\mathrm{ext}}>1$ the pressure of the reservoir increases, while the bottomhole pressure decreases with a negative slope.

\subsection{Similarities between Models with and without Influx Recharge}

In this section, we indicate a criteria to know when a drawdown curve is related to a reservoir with or without influx recharge. The analysis presented is necessary because del Angel et al. (2014) and Doublet and Blasingame (1995) mention that there is a similarity between a model of fluid flow in a single-porosity medium with influx recharge $\left(1 \phi-1 \kappa-q_{\mathrm{ext}} \neq 0\right)$ and a model of fluid flow in a closed double-porosity medium $\left(2 \phi-1 \kappa-q_{\mathrm{ext}}=0\right)$. However, a detailed analysis using the Bourdet derivative is not carried out in these works in order to elucidate this statement, which is based on the fact that both models have characteristic curves with a minimum from a transition stage. Indeed, in this regard, note that in Wang et al. (2017) are shown curves of a $3 \phi-1 \kappa-q_{\mathrm{ext}} \neq 0$ model that have a triple minimum in its drawdown derivative, i.e., those curves may have equivalence with the curves of a fluid flow in a closed quad-porosity medium. The discussion may also occur because despite the conceptual differences of the models being compared, their drawdown graphs are matched, as can be seen in Figs. 8(a) and 8(b). Therein, we compared the results from the following models: (a) $1 \phi-1 \kappa-q_{\mathrm{ext}} \neq 0$ vs $2 \phi-1 \kappa-q_{\mathrm{ext}}=0$ and (b) 

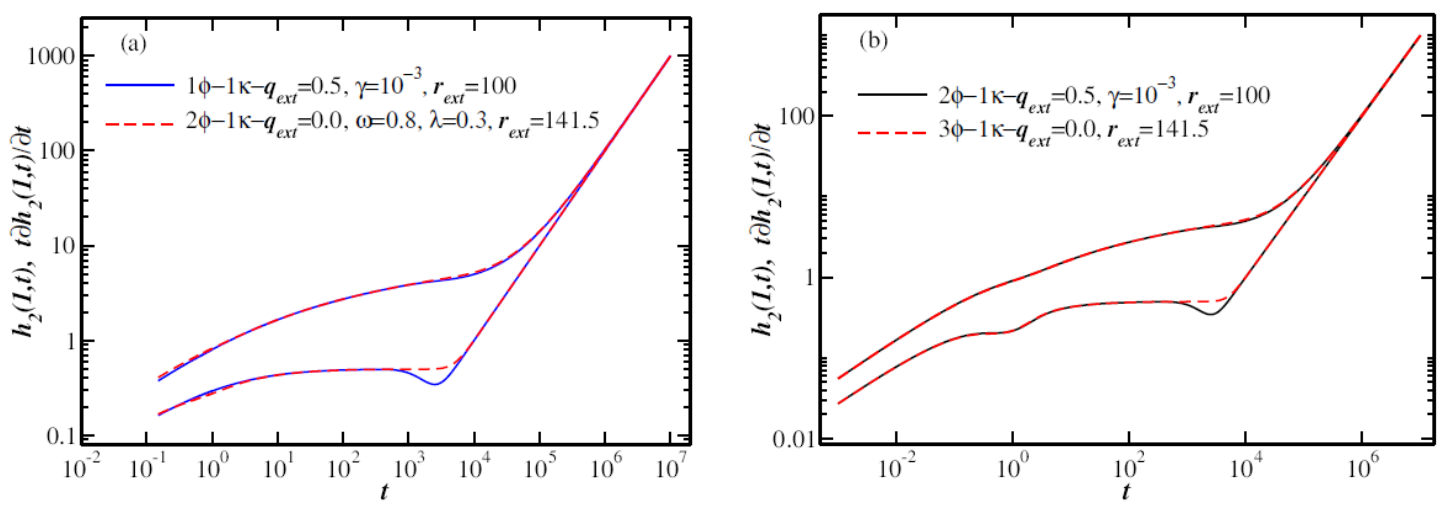

FIG. 8: Comparison of the drawdown pressure and its Bourdet derivative of the following models: (a) $1 \phi-1 \kappa-q_{\text {ext }} \neq 0$ vs $2 \phi-1 \kappa-q_{\mathrm{ext}}=0$ and (b) $2 \phi-1 \kappa-q_{\mathrm{ext}} \neq 0$ vs $3 \phi-1 \kappa-q_{\mathrm{ext}}=0$ (this triple-porosity model considers the differential equations (1)-(3) in Wu et al. (2007) together with the NN-BCs given in this work). As we can see in each frame, the difference between the drawdown curves are difficult to distinguish one of another. However, their Bourdet derivative show a clear difference in the transition period toward the outer boundary flow regime, i.e., in this transition period there is a minimum that depends on $q_{\text {ext }}$. The graph of the triple-porosity model is obtained with $\omega_{v}=0.05, \omega_{f}=0.4, \lambda_{f v}=0.005, \lambda_{f m}=0.5474$, and $\lambda_{v m}=0.1643(v$, $f$, and $m$ are for vuggs, fractures, and matrix, respectively), while the graph of the double porosity model with influx recharge is obtained with $\omega=0.4$ and $\lambda=0.5$.

$2 \phi-1 \kappa-q_{\mathrm{ext}} \neq 0$ vs $3 \phi-1 \kappa-q_{\mathrm{ext}}=0$. However, in the same figure it is seen that the Bourdet derivative exhibits a clear difference in the transition period toward the outer boundary flow regime (stage with a unit slope at long times). Even so, the question remains whether it is possible to obtain equivalent curves from the results of $1 \phi-1 \kappa-q_{\mathrm{ext}} \neq 0$ and $2 \phi-1 \kappa-q_{\mathrm{ext}}=0$ models.

In order to elucidate the differences between $1 \phi-1 \kappa-q_{\mathrm{ext}} \neq 0$ and $2 \phi-1 \kappa-q_{\mathrm{ext}}=0$ models, a comparison between both is shown in Fig. 9. This figure contains a characteristic drawdown curve (and/or its Bourdet derivative) of the $1 \phi-1 \kappa-q_{\mathrm{ext}} \neq 0$ model (see solid-black lines) in order to mark the differences with curves of the $2 \phi-1 \kappa-$ $q_{\text {ext }}=0$ model. Figure 9(a) shows drawdown curves with a monotonously increasing behavior, while the drawdown derivative has a minimum that increases its depth when the value of $q_{\mathrm{ext}}$ increases $\left(0 \leq q_{\mathrm{ext}}<1\right)$. In addition, a nonzero influx recharge leads to obtaining a minimum located just before the stage dominated by the outer boundary effect. The $2 \phi-1 \mathrm{~K}-q_{\mathrm{ext}}=0$ model also presents this latter stage, but its minimum, between the fractures and matrix flow regimes, is not necessarily located just before the beginning of the stage with a unit slope. This is exhibited in Figs. 9(b) and 9(c), in which the systematic effect of the parameters $\lambda$ and $\omega$ on drawdown derivatives is observed for this model. In both frames there are derivative curves with two minimums, i.e., these curves cannot be equivalent with the results in Fig. 9(a), which clearly has a minimum. The rest of the curves in Fig. 9(b) show behaviors which are dominated by the transition period between fractures and matrix flow regimes, which are present at short times when $\omega=0.05$ and at long times when $\omega=0.3$, see dashed-point and solid lines, respectively. Noticeably, these latter curves are very different from the ones in Fig. 9(a). Meanwhile, in Fig. 9(c), $\omega$ is varied for the purpose of obtaining a curve with a single minimum and with the restriction that the transition period, between the fractures and matrix flow regimes, remains just at the beginning of the stage with a unit slope. As we can see, we can not eliminate one of the minimums by varying $\omega$. For this reason, we solved a least-square minimization, where the results of the $1 \phi-1 \mathrm{\kappa}-q_{\mathrm{ext}} \neq 0$ model are taken as the input data, and the parametrized model is $2 \phi-1 \mathrm{\kappa}-q_{\mathrm{ext}}=0$. In this way, the curve-fitting problem leads to a value of $\omega=1$, i.e., the best fit is a curve of the $1 \phi-1 \kappa-q_{\mathrm{ext}}=0$ model [see red line with circles in Fig. 9(d)]. In addition, we realize another fit by considering the results of the $1 \phi-1 \kappa-q_{\text {ext }} \neq 0$ model as input data, which only are taken from the minimum and the stage with unit slope. The best-fitting curve reproduces the input data, but outside of the fitting interval the curve has another minimum, and at short times it is very different from the results of the $1 \phi-1 \kappa-q_{\text {ext }}=0$ model [see blue line with cross symbols in Fig. 9(d)]. The rest of the curves in Fig. 9(d) are given in order to show the behavior by varying $\omega$; they have two minimums.

Volume 23, Issue 10, 2020 

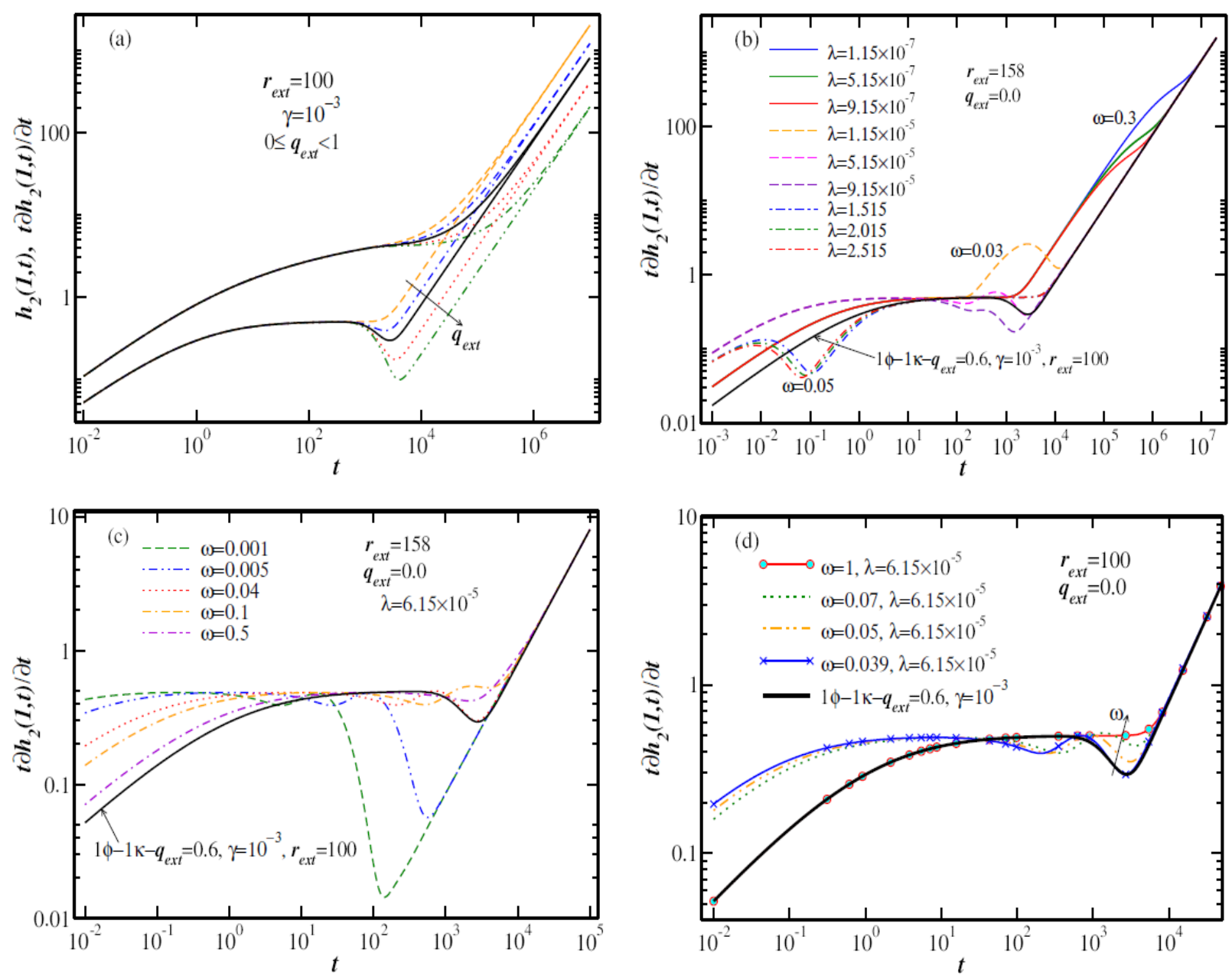

FIG. 9: In (a) are presented graphs of the $1 \phi-1 \kappa-q_{\mathrm{ext}} \neq 0$ model. The solid-black line remains as reference in (b), (c), and (d). The frames (b) and (c) show graphs of the drawdown derivative of the $2 \phi-1 \mathrm{~K}-q_{\text {ext }}=0$ model in order to exhibit the curve behaviors and their similarities with the curve of the $1 \phi-1 \kappa-q_{\text {ext }} \neq 0$ model. In (d) it is shown that the best-fitting curve of the $2 \phi-1 \kappa-q_{\text {ext }}=0$ model (red line with circle symbol) is a curve of the $1 \phi-1 \kappa-q_{\text {ext }}=0$ model, since $\omega=1$ is the result from the fitting.

Therefore, according to our analysis, it is impossible to obtain a quantitative equivalence between results of the $1 \phi-1 \kappa-q_{\mathrm{ext}} \neq 0$ and $2 \phi-1 \kappa-q_{\mathrm{ext}}=0$ models. We know that for the $1 \phi-1 \kappa-q_{\mathrm{ext}} \neq 0$ model, the only transition period is located at the beginning of the stage with unit slope. This stage has or does not have a minimum when there is or there is not an influx recharge, respectively. On the other hand, when there is a transition period that is not located at the beginning of the stage with unit slope, the porous medium is double-porosity, keeping out the possibility of quantitative equivalence with the $1 \phi-1 \kappa-q_{\mathrm{ext}} \neq 0$ model. However, note that when the only transition period is located at the beginning of the stage with unit slope, but the transition has two minimums, the reservoir has associated a double-porosity medium without influx recharge. This latter statement can be used as a criterion to distinguish real-life data from a double-porosity reservoir with closed boundary. By contrast, we remark that a single-porosity reservoir with influx recharge has a minimum at the beginning of the stage with unit slope.

It is worth mentioning that for simplicity we compare the $1 \phi-1 \kappa-q_{\mathrm{ext}} \neq 0$ and $2 \phi-1 \kappa-q_{\mathrm{ext}}=0$ models, on the understanding that similar conclusions must be obtained for other models, e.g., $2 \phi-1 \kappa-q_{\text {ext }} \neq 0$ vs $3 \phi-1 \kappa-q_{\mathrm{ext}}=0$. 


\section{CONCLUSIONS}

Using the joint Laplace-Hankel transform, we solved a fluid flow problem of interest in petroleum engineering and groundwater science. Our model describes the flow of a slightly compressible fluid in a double-porosity finite reservoir with Dirichlet-Dirichlet, Dirichlet-Neumann, Neumann-Dirichlet, and Neumann-Neumann boundary conditions. With this aim, the solution is divided into a stationary and a transient part. We validate the exact solution using the Stehfest method. In addition, when $\omega=1$ and $\lambda=0$, our formulas are reduced to those derived by other authors who have solved the equivalent problem for a single-porosity model (del Angel et al., 2014).

We find that the Cinelli formula [Eq. (24d)], related to NN-BCs, is incomplete because it does not include time-dependent terms, which impose the solution behavior at long time. For this reason, the results of Eq. (24d) are stationary always. The exact solution, in the limit of large $t$, is found by means of a series expansion in the Laplace space: thus, we identify the terms ignored by Eq. (24d), which are included in Eq. (36). On the other hand, regarding the DD-BCs, DN-BCs, and ND-BCs cases, we observed a correct convergence of solutions [Eqs. (24a)(24c)] along the domain of the solution, except in the inner boundary where a zero value is obtained. Furthermore, these solutions are oscillatory and slowly converging. We found that the inhomogeneous BCs are reproduced using the closed formulas derived in this work. In addition, these simplified formulas speed up the convergence of the solutions, compared to a direct use of the Cinelli relationships [Eq. (24)].

Finally, the characteristic behaviors of solutions (31), (33), (35), and (36) exhibit the different stages of flow at bottomhole: fracture-dominated, transitions-dominated, matrix-dominated, or recharge-dominated. The Bournet derivative, for the NN-BCs case, shows a minimum during the transition periods between the fractures and matrix flow regimes and the flow regime dominated by the influx recharge. This information can be used to give a criterion about whether the reservoir has recharge at the outer boundary at the same time that we can know the number of porosities associated with it. An extension of this work could include $2 \phi-2 \kappa$ models, as given by Lu et al. (2019b).

\section{DATA AVAILABILITY}

The data can be found on Mendeley Datasets with the following DOI: 10.17632/3837yfr46n.2.

\section{ACKNOWLEDGMENTS}

Luis X. Vivas-Cruz thanks CONACYT (Mexico) for its financial support through a PhD fellowship received during the realization of this work. Alfredo González-Calderón and Jorge Adrián Perera-Burgos acknowledge the support provided by CONACYT: Cátedras CONACYT para jóvenes investigadores. The authors acknowledge Felipe Pacheco-Vázquez, Wilberth Herrera, and Yarith del Angel for their comments and suggestions. Marco A. TanecoHernández acknowledges the support provided by SNI-CONACYT. Authors acknowledge the financial support provided by project FORDECYT No. 296327 Consolidación del Consorcio de Hidrocarburos, con Sede en Ciudad del Carmen, Campeche, México.

\section{REFERENCES}

Ahmed, T. and McKinney, P., Advanced Reservoir Engineering, Amsterdam: Elsevier, 2011.

Babak, P. and Azaiez, J., Unified Fractional Differential Approach for Transient Interporosity Flow in Naturally Fractured Media, Adv. Water Resour., vol. 74, pp. 302-317, 2014.

Boulton, N.S. and Streltsova, T.D., Unsteady Flow to a Pumped Well in a Fissured Water-Bearing Formation, J. Hydrol., vol. 35, nos. 3-4, pp. 257-270, 1977.

Bourdet, D., Well Test Analysis: the Use of Advanced Interpretation Models, vol. 3, Amsterdam: Elsevier, 2002.

Bourdet, D., Whittle, T.M., Douglas, A.A., and Pirard, Y.M., A New Set of Type Curves Simplifies Well-Test Analysis, World Oil, vol. 196, no. 6, pp. 95-106, 1983.

Bourdet, D., Ayoub, J.A., and Pirard, Y.M., Use of Pressure Derivative in Well Test Interpretation, Soc. Petrol. Eng. J., vol. 4, no. 2, pp. 293-302, 1989.

Volume 23, Issue 10, 2020 
Cai, J., Mirzaei-Paiaman, A., Vafai, K., and Wang, F., Preface: Theoretical and Mathematical Modeling of Flow and Transport in Porous Media, Special Topics Rev. Porous Media: Int. J., vol. 7, no. 2, pp. v-vii, 2016.

Camacho Velazquez, R., Gomez, S., Vasquez-Cruz, M.A., Fuenleal, N.A., Castillo, T., Ramos, G., Minutti, C., Mesejo, A., and Fuentes-Cruz, G., Well-Testing Characterization of Heavy-Oil Naturally Fractured Vuggy Reservoirs, SPE Heavy and Extra Heavy Oil Conference: Latin America, Society of Petroleum Engineers, 2014.

Carslaw, H.S. and Jaeger, J.C., Conduction of Heat in Solids, Oxford, UK: Oxford University Press, 1959.

Chen, Z.X., Transient Flow of Slightly Compressible Fluids through Double-Porosity, Double-Permeability Systems - A StateOf-The-Art Review, Transp. Porous Media, vol. 4, no. 2, pp. 147-184, 1989.

Chen, Z.X., Analytical Solutions for Double-Porosity, Double-Permeability and Layered Systems, J. Petrol. Sci. Eng., vol. 5, no. 1, pp. 1-24, 1990.

Cinelli, G., An Extension of the Finite Hankel Transform and Applications, Int. J. Eng. Sci., vol. 3, no. 5, pp. 539-559, 1965.

Clossman, P.J., An Aquifer Model for Fissured Reservoirs, Soc. Pet. Eng. J., vol. 15, no. 5, pp. 385-398, 1975.

Da Prat, G., Well Test Analysis for Fractured Reservoir Evaluation, vol. 27, New York: Elsevier, 1990.

De Smedt, F., Analytical Solution for Constant-Rate Pumping Test in Fissured Porous Media with Double-Porosity Behaviour, Transp. Porous Media, vol. 88, no. 3, pp. 479-489, 2011.

Debnath, L. and Bhatta, D., Integral Transforms and Their Applications, London, New York: CRC Press, 2014.

del Angel, Y., Nuñez López, M., and Velasco-Hernández, J.X., Pressure Transient Analysis with Exponential and Power Law Boundary Flux, J. Petrol. Sci. Eng., vol. 121, pp. 149-158, 2014.

Doublet, L.E. and Blasingame, T.A., Decline Curve Analysis Using Type Curves: Water Influx/Waterflood Cases, Waterflood Cases, paper SPE 30774 presented at the 1995 Annual Technical Conf. and Exhibition, Dallas, Texas, pp. 1-23, 1995.

Ehlig-Economides, C.A. and Joseph, J., A New Test for Determination of Individual Layer Properties in a Multilayered Reservoir, SPE Form. Eval., vol. 2, no. 3, pp. 261-283, 1987.

Goltz, M.N. and Oxley, M.E., Analytical Modeling of Aquifer Decontamination by Pumping when Transport Is Affected by Rate-Limited Sorption, Water Resour. Res., vol. 27, no. 4, pp. 547-556, 1991.

Gomes, E. and Ambastha, A.K., An Analytical Pressure-Transient Model for Multilayered, Composite Reservoirs with Pseudosteady-State Formation Crossflow, SPE Western Regional Meeting, Society of Petroleum Engineers, pp. 221-233, 1993.

González-Calderón, A., Vivas-Cruz, L.X., and Salmerón-Rodríguez, U., Exact Analytical Solution of the Telegraphic Warren and Root Model, Transp. Porous Media, vol. 120, no. 2, pp. 433-448, 2017.

Greenberg, M.D., Advanced Engineering Mathematics, Upper Saddle River, NJ: Prentice-Hall, 1998.

Gringarten, A.C., From Straight Lines to Deconvolution: The Evolution of the State of the Art in Well Test Analysis, SPE Reservoir Eval. Eng., vol. 11, no. 1, pp. 41-62, 2008.

Hurst, W., Unsteady Flow of Fluids in Oil Reservoirs, Phys., vol. 5, no. 1, pp. 20-30, 1934.

Javandel, I. and Witherspoon, P.A., Analytical Solution of a Partially Penetrating Well in a Two-Layer Aquifer, Water Resour. Res., vol. 19, no. 2, pp. 567-578, 1983.

Jiang, Q. and Gao, C., On the General Expressions of Finite Hankel Transform, Sci. China Phys. Mech., vol. 53, no. 11, pp. 21252130, 2010.

Ju, B., Mathematical Model and Analytical Solutions for Unsteady Flow in Natural Gas Reservoirs, J. Porous Media, vol. 17, no. 4, pp. 279-285, 2014.

Katz, M.L. and Tek, M.R., A Theoretical Study of Pressure Distribution and Fluid Flux in Bounded Stratified Porous Systems with Crossflow, Soc. Petrol. Eng. J., vol. 2, no. 1, pp. 68-82, 1962.

Kruseman, G.P., De Ridder, N.A., and Verweij, J.M., Analysis and Evaluation of Pumping Test Data, 2nd Ed., The Netherlands: International Institute for Land Reclamation and Improvement, 1994.

Kuhlman, K.L., Malama, B., and Heath, J.E., Multiporosity Flow in Fractured Low-Permeability Rocks, Water Resour. Res., vol. 51, no. 2, pp. 848-860, 2015.

Liu, M.X. and Chen, Z.X., Exact Solution for Flow of Slightly Compressible Fluids through Multiple-Porosity, MultiplePermeability Media, Water Resour. Res., vol. 26, no. 7, pp. 1393-1400, 1990.

Lu, J., Shi, S., and Rahman, M.M., New Mathematical Models for Production Performance of a Well Producing at Constant 
Bottomhole Pressure, Special Topics Rev. Porous Media: Int. J., vol. 9, no. 3, pp. 261-278, 2018.

Lu, J., Owayed, J.F., Xu, J., and Rahman, M.M., An Analytical Model on Production Performance of Multiple Wells Producing at Constant Bottomhole Pressures, Special Topics Rev. Porous Media: Int. J., vol. 10, no. 1, pp. 31-48, 2019a.

Lu, J., Qu, J., and Rahman, M.M., A New Dual-Permeability Model for Naturally Fractured Reservoirs, Special Topics Rev. Porous Media: Int. J., vol. 10, no. 5, pp. 485-502, 2019b.

Matthews, C.S. and Russell, D.G., Pressure Buildup and Flow Tests in Wells, vol. 1, Richardson, Texas: Society of Petroleum Engineers, 1967.

Mavor, M.J. and Cinco-Ley, H., Transient Pressure Behavior of Naturally Fractured Reservoirs, SPE California Regional Meeting, Society of Petroleum Engineers, 1979.

Moench, A.F., Convergent Radial Dispersion in a Double-Porosity Aquifer with Fracture Skin: Analytical Solution and Application to a Field Experiment in Fractured Chalk, Water Resour. Res., vol. 31, no. 8, pp. 1823-1835, 1995.

Muskat, M., The Flow of Compressible Fluids through Porous Media and Some Problems in Heat Conduction, Phys., vol. 5, no. 3, pp. 71-94, 1934.

Nie, R.S., Meng, Y.F., Jia, Y.L., Zhang, F.X., Yang, X.T., and Niu, X.N., Dual Porosity and Dual Permeability Modeling of Horizontal Well in Naturally Fractured Reservoir, Transp. Porous Media, vol. 92, no. 1, pp. 213-235, 2012.

Ozkan, E. and Raghavan, R., Some New Solutions to Solve Problems in Well Test Analysis: Part 1-Analytical Considerations, SPE J., pp. 1-63, 1988.

Poularikas, A.D., Transforms and Applications Handbook, London, New York: CRC Press, 2010.

Prats, M., Interpretation of Pulse Tests in Reservoirs with Crossflow between Contiguous Layers, SPE Form. Eval., vol. 1, no. 5, pp. 511-520, 1986.

Russell, D.G. and Prats, M., Performance of Layered Reservoirs with Crossflow-Single-Compressible-Fluid Case, Soc. Petrol. Eng. J., vol. 2, no. 1, pp. 53-67, 1962.

Shah, P.C. and Thambynayagam, R.K.M., Transient Pressure Response of a Well with Partial Completion in a Two-Layer Crossflowing Reservoir, SPE Annual Technical Conf. and Exhibition, Society of Petroleum Engineers, pp. 213-225, 1992.

Shanks, D., Non-Linear Transformations of Divergent and Slowly Convergent Sequences, Stud. Appl. Math., vol. 34, nos. 1-4, pp. 1-42, 1955.

Singhal, B.B.S. and Gupta, R.P., Applied Hydrogeology of Fractured Rocks, New York: Springer Science \& Business Media, 2010. Sneddon, I.N., On Finite Hankel Transforms, London, Edinburgh Dublin Philos. Mag. J. Sci., vol. 37, no. 264, pp. 17-25, 1946.

Stehfest, H., Algorithm 368: Numerical Inversion of Laplace Transforms [D5], Commun. ACM, vol. 13, no. 1, pp. 47-49, 1970.

Tong, D. and Hu, H., Flow Analysis of Non-Newtonian Viscoelastic Fluids in Porous Media, J. Porous Media, vol. 13, no. 5, pp. 477-486, 2010.

Uldrich, D.O. and Ershaghi, I., A Method for Estimating the Interporosity Flow Parameter in Naturally Fractured Reservoirs, Soc. Petrol. Eng. J., vol. 19, no. 5, pp. 324-332, 1979.

van Everdingen, A.F. and Hurst, W., The Application of the Laplace Transformation to Flow Problems in Reservoirs, J. Petrol. Technol., vol. 1, no. 12, pp. 305-324, 1949.

Wang, D., Yao, J., Cai, M., and Liu, P., Transient Pressure and Productivity Analysis in Carbonate Geothermal Reservoirs with Changing External Boundary Flux, Therm. Sci., vol. 21, no. 1, pp. S177-S184, 2017.

Wang, X. and Gong, Y., An Elastodynamic Solution for Multilayered Cylinders, Int. J. Eng. Sci., vol. 30, no. 1, pp. 25-33, 1992.

Warren, J.E. and Root, P.J., The Behavior of Naturally Fractured Reservoirs, Soc. Petrol. Eng. J., vol. 3, no. 3, pp. $245-255,1963$.

Wu, Y.S., An Approximate Analytical Solution for Non-Darcy Flow Toward a Well in Fractured Media, Water Resour. Res., vol. 38, no. 3, pp. 1-7, 2002.

Wu, Y.S., Ehlig-Economides, C., Qin, G., Kang, Z., Zhang, W., Ajayi, B., and Tao, Q., A Triple-Continuum Pressure-Transient Model for a Naturally Fractured Vuggy Reservoir, SPE J., 2007.

Xi, W. and Yuning, G., A Theoretical Solution for Axially Symmetric Problems in Elastodynamics, Acta Mech. Sin.-PRC, vol. 7, no. 3, pp. 275-282, 1991.

Yao, Y., Wu, Y.S., and Zhang, R., The Transient Flow Analysis of Fluid in a Fractal, Double-Porosity Reservoir, Transp. Porous Media, vol. 94, no. 1, pp. 175-187, 2012.

Volume 23, Issue 10, 2020 
Young, R., Pressure Transients in a Double-Porosity Medium, Water Resour. Res., vol. 28, no. 5, pp. 1261-1270, 1992.

Zhang, X. and Tong, D., A Generalized Weber Transform and Its Inverse Formula, Appl. Math. Comput., vol. 193, no. 1, pp. 116126, 2007.

Zhou, Q., Oldenburg, C.M., and Rutqvist, J., Revisiting the Analytical Solutions of Heat Transport in Fractured Reservoirs Using a Generalized Multirate Memory Function, Water Resour. Res., vol. 55, no. 2, pp. 1405-1428, 2019.

\section{APPENDIX A. EXACT SOLUTIONS}

In this Appendix, we present additional details of the procedure developed in this work to solve the partial differential equation of the studied model.

The finite Hankel transform of a well-behaved function $h(r)$ is expressed as follows (Cinelli, 1965):

$$
\tilde{h}\left(k_{i}\right)=H[h(r)]=\int_{a}^{b} r h(r) K\left(r, k_{i}, a\right) d r
$$

where $K\left(r, k_{i}, a\right)$ is a kernel that depends on the inner $\mathrm{BC}$ :

$$
\begin{aligned}
K\left(r, k_{i}, a\right) & =J_{0}\left(k_{i} r\right) Y_{0}\left(k_{i} a\right)-J_{0}\left(k_{i} a\right) Y_{0}\left(k_{i} r\right), & & \text { for DD and DN-BCs } \\
& =J_{1}\left(k_{i} a\right) Y_{0}\left(k_{i} r\right)-J_{0}\left(k_{i} r\right) Y_{1}\left(k_{i} a\right), & & \text { for ND and NN-BCs. }
\end{aligned}
$$

There is also an equivalent kernel that depends only on the outer boundary. Using the previous definition, the finite Hankel transform of the Laplacian is given by (Cinelli, 1965):

$$
\begin{aligned}
H\left[\frac{d^{2} h}{d r^{2}}+\frac{1}{r} \frac{d h}{d r}\right] & =\frac{2}{\pi} \frac{J_{0}\left(k_{i} a\right)}{J_{0}\left(k_{i} b\right)} h(b)-\frac{2}{\pi} h(a)-k_{i}^{2} \tilde{h}\left(k_{i}\right), & & \text { for DD-BCs, } \\
& =-\frac{2}{\pi k_{i}} \frac{J_{0}\left(k_{i} a\right)}{J_{1}\left(k_{i} b\right)} h^{\prime}(b)-\frac{2}{\pi} h(a)-k_{i}^{2} \tilde{h}\left(k_{i}\right), & & \text { for DN-BCs, } \\
& =-\frac{2}{\pi k_{i}} h^{\prime}(a)-\frac{2 J_{1}\left(k_{i} a\right)}{\pi J_{0}\left(k_{i} b\right)} h(b)-k_{i}^{2} \tilde{h}\left(k_{i}\right), & & \text { for ND-BCs, } \\
& =\frac{2 J_{1}\left(k_{i} a\right)}{\pi k_{i} J_{1}\left(k_{i} b\right)} h^{\prime}(b)-\frac{2}{\pi k_{i}} h^{\prime}(a)-k_{i}^{2} \tilde{h}\left(k_{i}\right), & & \text { for NN-BCs. }
\end{aligned}
$$

where $h^{\prime}(a)$ is the derivative of $h(r)$ evaluated in $r=a$. These latter expressions are used to obtain the inverse finite Hankel transform given in Eq. (24).

\section{A.1 Finite Reservoir with Dirichlet-Dirichlet Boundary Conditions}

Using the JLHT to obtain the solution of a fluid flow in a finite reservoir with constant pressure in both boundaries is very simple. From Eq. (23a) and Eqs. (15) and (17), we get

$$
\tilde{\widehat{h}}_{2}\left(k_{i}, s\right)=-\frac{1}{s} \mathcal{F}\left(k_{i}, s\right),
$$

where

$$
\mathcal{F}\left(k_{i}, s\right)=\frac{2}{\pi\left[\eta(s)+k_{i}^{2}\right]},
$$

$\eta(s)$ where is defined in Eq. (22).

Taking the inverse Laplace transform of Eq. (A.4) leads to the following expression in Hankel space:

$$
\tilde{h}_{2}\left(k_{i}, t\right)=-\frac{2}{\pi k_{i}^{2}}+\tilde{g}\left(k_{i}, t\right),
$$


where

$$
\tilde{g}\left(k_{i}, t\right)=\frac{1}{v}\left[(\nu+\varrho) \exp \left\{-\frac{\xi+v}{2 \psi} t\right\}+(v-\varrho) \exp \left\{-\frac{\xi-v}{2 \psi} t\right\}\right]
$$

and

$$
\begin{aligned}
& \psi=\omega(\omega-1), \\
& \xi=-\lambda+k_{i}^{2}(\omega-1), \\
& \varrho=\lambda+k_{i}^{2}(\omega-1), \\
& \nu=\sqrt{\xi^{2}+4 k_{i}^{2} \lambda \psi} .
\end{aligned}
$$

Because the term $-2 / \pi k_{i}^{2}$ is not related to time, its finite inverse Hankel transform is the stationary solution of model (3). Therefore, we replace the inversion of $-2 / \pi k_{i}^{2}$ with the stationary solution of the Laplace equation, $\nabla^{2} h_{2}=0$, in cylindrical coordinates. Namely, the following equality holds true:

$$
1-\frac{\log (r)}{\log \left(r_{\mathrm{ext}}\right)}=-\pi \sum_{i=1}^{\infty} \frac{\mathcal{I}_{0,0}\left(k_{i}, r, 1\right) J_{0}^{2}\left(r_{\mathrm{ext}} k_{i}\right)}{J_{0}^{2}\left(k_{i}\right)-J_{0}^{2}\left(r_{\mathrm{ext}} k_{i}\right)} .
$$

Substituting Eq. (A.6) into Eq. (24) for the DD-BCs case, and simplifying with the previous closed formula, the exact solution is

$$
h_{2}(r, t)=1-\frac{\log (r)}{\log \left(r_{\mathrm{ext}}\right)}+\frac{\pi}{2} \sum_{i=1}^{\infty} \frac{\tilde{g}\left(k_{i}, t\right) \mathcal{I}_{0,0}\left(k_{i}, r, 1\right) J_{0}^{2}\left(r_{\mathrm{ext}} k_{i}\right)}{J_{0}^{2}\left(k_{i}\right)-J_{0}^{2}\left(r_{\mathrm{ext}} k_{i}\right)},
$$

where the $k_{i}$ are the positive roots of $\mathcal{I}_{0,0}\left(k_{i}, 1, r_{\text {ext }}\right)=0$.

The flux is obtained by substituting Eq. (31) into Eq. (8). This is accomplished as follows:

$$
j_{2}(t)=\frac{1}{\log \left(r_{\mathrm{ext}}\right)}-\frac{\pi}{2} \sum_{i=1}^{\infty} \frac{k_{i} \tilde{g}\left(k_{i}, t\right) \mathcal{I}_{0,1}\left(k_{i}, 1,1\right) J_{0}^{2}\left(r_{\mathrm{ext}} k_{i}\right)}{J_{0}^{2}\left(k_{i}\right)-J_{0}^{2}\left(r_{\mathrm{ext}} k_{i}\right)} .
$$
taken.

Equations (31) and (32) recover the formulas in Muskat (1934) when the limit of single-porosity medium is

\section{A.2 Finite Reservoir with Dirichlet-Neumann Boundary Conditions}

Fluid flow in a reservoir with constant pressure at the bottomhole and influx recharge $f(t)$ at the outer boundary is considered. The influx function $f(t)$ is given in Eq. (12). Substituting Eqs. (15) and (18) into Eq. (23b) leads to the following formula:

$$
\tilde{\widehat{h}}_{2}\left(k_{i}, s\right)=\left(\frac{q_{\mathrm{ext}} J_{0}\left(k_{i}\right)}{r_{\mathrm{ext}} k_{i} J_{1}\left(r_{\mathrm{ext}} k_{i}\right)} \frac{1}{\left(\gamma s^{2}+s\right)}-\frac{1}{s}\right) \mathcal{F}\left(k_{i}, s\right) .
$$

Taking the inverse Laplace transform of Eq. (A.12), we obtain

$$
\tilde{h}_{2}\left(k_{i}, t\right)=\frac{2}{\pi k_{i}^{2}}\left[\frac{q_{\mathrm{ext}}}{r_{\mathrm{ext}}} \frac{J_{0}\left(k_{i}\right)}{k_{i} J_{1}\left(r_{\mathrm{ext}} k_{i}\right)}-1\right]+\tilde{g}\left(k_{i}, t\right),
$$

where

$$
\begin{aligned}
\tilde{g}\left(k_{i}, t\right) & =\frac{q_{\mathrm{ext}} J_{0}\left(k_{i}\right)}{\pi k_{i}^{3} r_{\mathrm{ext}} J_{1}\left(r_{\mathrm{ext}} k_{i}\right) \vartheta v} \exp \left\{-\frac{\xi+v}{2 \psi} t\right\}\left[\left(-2 k_{i}^{2} \gamma(\gamma \lambda+\omega-1) \exp \left\{\left(\frac{\xi+v}{2 \psi}-\frac{1}{\gamma}\right) t\right\}\right.\right. \\
& \left.\left.+(\psi+\lambda \gamma)\left(\exp \left\{\frac{v}{\psi} t\right\}+1\right)\right) v+\left(\lambda \gamma\left(\xi-2 k_{i}^{2} \psi\right)-\psi \varrho\right)\left(\exp \left\{\frac{v}{\psi} t\right\}-1\right)\right] \\
& -\frac{1}{\pi k_{i}^{2} v} \exp \left\{-\frac{\xi+v}{2 \psi} t\right\}\left[\left(\exp \left\{\frac{v}{\psi} t\right\}-1\right) \varrho-\left(\exp \left\{\frac{v}{\psi} t\right\}+1\right) v\right],
\end{aligned}
$$


and $\xi, \psi, \varrho$, and $v$ are given in Eq. (A.8). In addition, we have

$$
\vartheta=\gamma \xi-\psi+k_{i}^{2} \gamma^{2} \lambda \text {. }
$$

Similar to DD-BCs case, Eq. (A.13) has terms not related to time, whose inverse finite Hankel transform is the stationary solution of model (3). Therefore, this inverse is equal to the solution of the Laplace equation with DN-BCs

$$
1-q_{\mathrm{ext}} \log (r)=\pi \sum_{i=1}^{\infty}\left(\frac{q_{\mathrm{ext}}}{r_{\mathrm{ext}}} \frac{J_{0}\left(k_{i}\right)}{k_{i} J_{1}\left(r_{\mathrm{ext}} k_{i}\right)}-1\right) \frac{\mathcal{I}_{0,0}\left(k_{i}, r, 1\right) J_{1}^{2}\left(r_{\mathrm{ext}} k_{i}\right)}{J_{0}^{2}\left(k_{i}\right)-J_{1}^{2}\left(r_{\mathrm{ext}} k_{i}\right)} .
$$

Substituting Eq. (A.13) into Eq. (24b), and simplifying with the closed formula (A.16), the exact solution is

$$
h_{2}(r, t)=1-q_{\mathrm{ext}} \log (r)+\frac{\pi^{2}}{2} \sum_{i=1}^{\infty} \frac{k_{i}^{2} \tilde{g}\left(k_{i}, t\right) \mathcal{I}_{0,0}\left(k_{i}, r, 1\right) J_{1}^{2}\left(r_{\mathrm{ext}} k_{i}\right)}{J_{0}^{2}\left(k_{i}\right)-J_{1}^{2}\left(r_{\mathrm{ext}} k_{i}\right)},
$$

where the $k_{i}$ are the positive roots of $\mathcal{I}_{1,0}\left(k_{i}, r_{\mathrm{ext}}, 1\right)=0$.

In addition, from Eqs. (8) and (33), the flux at the bottomhole can be written as

$$
j_{2}(t)=q_{\mathrm{ext}}-\frac{\pi^{2}}{2} \sum_{i=1}^{\infty} \frac{k_{i}^{3} \tilde{g}\left(k_{i}, t\right) \mathcal{I}_{0,1}\left(k_{i}, 1,1\right) J_{1}^{2}\left(r_{\mathrm{ext}} k_{i}\right)}{J_{0}^{2}\left(k_{i}\right)-J_{1}^{2}\left(r_{\mathrm{ext}} k_{i}\right)} .
$$

Equations (33) and (34) recover the formulas in Muskat (1934) and Hurst (1934) when the limit of single-porosity is taken.

\section{A.3 Finite Reservoir with Neumann-Dirichlet Boundary Conditions}

Assuming a constant terminal rate at the bottomhole and constant pressure at the outer boundary of a finite reservoir, the pressure is found; i.e., the ND-BCs case is solved. Substituting Eqs. (16) and (17) in Eq. (23c), we obtain

$$
\tilde{\hat{h}}_{2}\left(k_{i}, s\right)=\frac{1}{k_{i} s} \mathcal{F}\left(k_{i}, s\right) .
$$

Taking the inverse Laplace transform of Eq. (A.19) leads to

$$
\tilde{h}_{2}\left(k_{i}, t\right)=\frac{2}{\pi k_{i}^{3}}+\tilde{g}\left(k_{i}, t\right)
$$

where

$$
\tilde{g}\left(k_{i}, t\right)=\frac{1}{v}\left[(\varrho-v) \exp \left\{\frac{v-\xi}{2 \psi} t\right\}-(\varrho+v) \exp \left\{-\frac{\xi+v}{2 \psi} t\right\}\right] .
$$

From the inverse of terms not related to time in Eq. (A.20) and from the time-independent solution of the model (3), we have

$$
\log \left(\frac{r_{\mathrm{ext}}}{r}\right)=\pi \sum_{i=1}^{\infty} \frac{\mathcal{I}_{1,0}\left(k_{i}, 1, r\right) J_{0}^{2}\left(r_{\mathrm{ext}} k_{i}\right)}{k_{i}\left[J_{1}^{2}\left(k_{i}\right)-J_{0}^{2}\left(r_{\mathrm{ext}} k_{i}\right)\right]} .
$$

Placing Eq. (A.20) in Eq. (24c), and using Eq. (A.22), the exact solution is given by

$$
h_{2}(r, t)=\log \left(\frac{r_{\mathrm{ext}}}{r}\right)+\frac{\pi}{2} \sum_{i=1}^{\infty} \frac{\tilde{g}\left(k_{i}, t\right) \mathcal{I}_{1,0}\left(k_{i}, 1, r\right) J_{0}^{2}\left(r_{\mathrm{ext}} k_{i}\right)}{k_{i}\left[J_{1}^{2}\left(k_{i}\right)-J_{0}^{2}\left(r_{\mathrm{ext}} k_{i}\right)\right]},
$$

where the $k_{i}$ are the positive roots of $\mathcal{I}_{1,0}\left(k_{i}, 1, r_{\text {ext }}\right)=0$.

Equation (35) recovers the formulas in Matthews and Russell (1967) when the limit of single-porosity is taken. 


\section{A.4 Finite Reservoir with Neumann-Neumann Boundary Conditions}

The pressure of a fluid in a double-porosity reservoir with constant terminal rate and influx recharge $f(t)$ at the outer boundary is given. Replacing Eqs. (16) and (18) in Eq. (23d), we obtain:

$$
\begin{aligned}
\tilde{\hat{h}}_{2}\left(k_{i}, s\right) & =\frac{\mathcal{F}\left(k_{i}, s\right)}{k_{i} s}-q_{\mathrm{ext}} \frac{J_{1}\left(k_{i}\right) \mathcal{F}\left(k_{i}, s\right)}{r_{\mathrm{ext}} k_{i}\left(\gamma s^{2}+s\right) J_{1}\left(r_{\mathrm{ext}} k_{i}\right)}, \\
& =\widetilde{\widehat{h}}_{2, \mathrm{nf}}+\widetilde{\widehat{h}}_{2, \mathrm{f}}
\end{aligned}
$$

The no-flow term $\tilde{\widehat{h}}_{2, \mathrm{nf}}$ has the following inverse Laplace transform:

$$
\tilde{h}_{2, \mathrm{nf}}\left(k_{i}, t\right)=\frac{2}{\pi k_{i}^{3}}+\tilde{g}\left(k_{i}, t\right)
$$

where $\tilde{g}\left(k_{i}, t\right)$ is equal to the expression in Eq. (A.21), with the difference that the values of $k_{i}$ comes from the positive roots of $\mathcal{I}_{1,1}\left(k_{i}, 1, r_{\text {ext }}\right)$.

The second term in the right hand side (RHS) of Eq. (A.24) is an influx term, whose inverse Laplace transform is found using the convolution theorem. This inverse can be written as

$$
\begin{aligned}
\tilde{h}_{2, \mathrm{f}} & =\frac{1}{r_{\mathrm{ext}}} \tilde{\mathcal{G}}\left(k_{i}\right)\left(\mathcal{L}^{-1}\{\widehat{f}(s)\} * \mathcal{L}^{-1}\left\{\tilde{\widehat{u}}\left(k_{i}, s\right)\right\}\right), \\
& =\frac{\tilde{\mathcal{G}}\left(k_{i}\right)}{r_{\mathrm{ext}}} \int_{0}^{t} f(t-\zeta) \tilde{u}\left(k_{i}, \zeta\right) d \zeta \\
& =-\frac{q_{\mathrm{ext}}}{r_{\mathrm{ext}}} \tilde{\mathcal{G}}\left(k_{i}\right)\left[\tilde{Q}_{1}\left(k_{i}, t\right)-\tilde{Q}_{2}\left(k_{i}, t\right)\right]
\end{aligned}
$$

in which we use the following inverse Laplace transform:

$$
\tilde{u}\left(k_{i}, t\right)=\frac{1}{2 \omega \nu} \exp \left\{-\frac{\xi+v}{2 \psi} t\right\}\left[\left(1-\exp \left\{\frac{v}{\psi} t\right\}\right)(\xi+2 \omega \lambda)+\left(1+\exp \left\{\frac{v}{\psi} t\right\}\right) v\right] .
$$

In previous equations, we have

$$
\begin{aligned}
\tilde{\mathcal{G}}\left(k_{i}\right) & =\frac{2 J_{1}\left(k_{i}\right)}{\pi k_{i} J_{1}\left(r_{\text {ext }} k_{i}\right)}, \\
\tilde{u}\left(k_{i}, s\right) & =\frac{1}{\eta(s)+k_{i}^{2}}, \\
\tilde{Q}_{1}\left(k_{i}, t\right) & =\int_{0}^{t} \tilde{u}\left(k_{i}, \zeta\right) d \zeta \\
& =\frac{1}{2 k_{i}^{2} v} \exp \left\{-\frac{(\xi+v)}{2 \psi} t\right\}\left[\left(\exp \left\{\frac{v}{\psi} t\right\}-1\right) \varrho+\left(2 \exp \left\{\frac{(\xi+v)}{2 \psi} t\right\}-\exp \left\{\frac{v}{\psi} t\right\}-1\right) v\right] \\
\tilde{Q}_{2}\left(k_{i}, t\right) & =\int_{0}^{t} \mathrm{e}^{-(t-\zeta) / \gamma} \tilde{u}\left(k_{i}, \zeta\right) d \zeta \\
& =\frac{\gamma(\omega-1)}{v} \mathrm{e}^{-t / \gamma}\left[\frac{\mathcal{A}+v}{\mathcal{B}-2 \psi}-\frac{\mathcal{A}-v}{\mathcal{C}-2 \psi}-\mathrm{e}^{-(\mathcal{B}-2 \psi) /(2 \gamma \psi) t}\left(\frac{\mathrm{e}^{(\nu / \psi) t}(\mathcal{A}-v)}{\mathcal{C}+2 \psi}+\frac{\mathcal{A}+v}{\mathcal{B}-2 \psi}\right)\right]
\end{aligned}
$$

where $\xi, \nu$, and $\psi$ are given in Eq. (A.8), $\mathcal{A}=\xi+2 \omega \lambda, \mathcal{B}=(\nu+\xi) \gamma$, and $\mathcal{C}=(\nu-\xi) \gamma$. 
Therefore, the inverse Laplace transform of Eq. (A.24) is the sum of Eqs. (A.25) and (A.26):

$$
\tilde{h}_{2}\left(k_{i}, t\right)=\tilde{h}_{2, \mathrm{nf}}+\tilde{h}_{2, \mathrm{f}} .
$$

Since in the NN-BCs case the Laplace equation has no solution, the asymptotic solution for long time is found by means of a series expansion of the solution in Laplace space, i.e., the expansion of Eq. (43) about $s=0$ (Prats, 1986; van Everdingen and Hurst, 1949) is developed. Thus, the behavior for long time of the no-flow term was given by van Everdingen and Hurst (1949):

$$
h_{2, \mathrm{n}-\mathrm{f}}(r, t)=\frac{2}{r_{\mathrm{ext}}^{2}-1}\left(\frac{r^{2}}{4}+t\right)-\frac{r_{\mathrm{ext}}^{2}}{r_{\mathrm{ext}}^{2}-1} \log (r)-\frac{3 r_{\mathrm{ext}}^{4}-4 r_{\mathrm{ext}}^{4} \log \left(r_{\mathrm{ext}}\right)-2 r_{\mathrm{ext}}^{2}-1}{4\left(r_{\mathrm{ext}}^{2}-1\right)^{2}},
$$

while the influx term was found by del Angel et al. (2014):

$$
h_{2, \mathrm{f}}(r, t)=q_{\mathrm{ext}}\left[\frac{2}{r_{\mathrm{ext}}^{2}-1}\left(\frac{r^{2}}{4}+t\right)-\frac{\log (r)}{r_{\mathrm{ext}}^{2}-1}-\frac{r_{\mathrm{ext}}^{4}+2 r_{\mathrm{ext}}^{2}-4 r_{\mathrm{ext}}^{2} \log \left(r_{\mathrm{ext}}\right)-3}{4\left(r_{\mathrm{ext}}^{2}-1\right)^{2}}\right]+\frac{2 \gamma q_{\mathrm{ext}}\left(1-\mathrm{e}^{-t / \gamma}\right)}{r_{\mathrm{ext}}^{2}-1} .
$$

Equations (A.30) and (A.31) come from studies of fluid flow in a single-porosity medium. However, they can be used in the solution of the double-porosity model since at long time the fluid behavior resembles that of a fluid in homogeneous reservoir. Mathematically,

$$
\eta(s) \rightarrow s \quad \text { as } \quad s \rightarrow 0,
$$

which is the $\eta(s)$ of a single-porosity medium. Accordingly, we can use Eqs. (A.30) and (A.31) in the model of double-porosity.

Equations (A.20) and (A.26) include the terms $2 / \pi k_{i}^{3}$ and $-q_{\text {ext }} \tilde{\mathcal{G}}\left(k_{i}\right) /\left(r_{\text {ext }} k_{i}^{2}\right)$, respectively, whose inverse finite Hankel transform is time-independent. This implies that the time-independent terms of Eqs. (A.30) and (A.31) must equal the inverse finite Hankel transform of $2 / \pi k_{i}^{3}$ and $-q_{\text {ext }} \tilde{\mathcal{G}}\left(k_{i}\right) /\left(r_{\text {ext }} k_{i}^{2}\right)$, respectively. Therefore,

$$
\pi \sum_{i=1}^{\infty} \frac{\mathcal{I}_{1,0}\left(k_{i}, 1, r\right) J_{1}^{2}\left(r_{\mathrm{ext}} k_{i}\right)}{k_{i}\left[J_{1}^{2}\left(k_{i}\right)-J_{1}^{2}\left(r_{\mathrm{ext}} k_{i}\right)\right]}=\frac{r^{2}}{2\left(r_{\mathrm{ext}}^{2}-1\right)}-\frac{r_{\mathrm{ext}}^{2}}{r_{\mathrm{ext}}^{2}-1} \log (r)-\frac{3 r_{\mathrm{ext}}^{4}-4 r_{\mathrm{ext}}^{4} \log \left(r_{\mathrm{ext}}\right)-2 r_{\mathrm{ext}}^{2}-1}{4\left(r_{\mathrm{ext}}^{2}-1\right)^{2}},
$$

and

$$
\begin{aligned}
& -q_{\mathrm{ext}} \pi \sum_{i=1}^{\infty} \frac{J_{1}\left(k_{i}\right) \mathcal{I}_{1,0}\left(k_{i}, 1, r\right) J_{1}\left(r_{\mathrm{ext}} k_{i}\right)}{r_{\mathrm{ext}} k_{i}\left[J_{1}^{2}\left(k_{i}\right)-J_{1}^{2}\left(r_{\mathrm{ext}} k_{i}\right)\right]}=-q_{\mathrm{ext}}\left[\frac{r^{2}}{2\left(r_{\mathrm{ext}}^{2}-1\right)}-\frac{\log (r)}{r_{\mathrm{ext}}^{2}-1}\right. \\
& \left.-\frac{r_{\mathrm{ext}}^{4}+2 r_{\mathrm{ext}}^{2}-4 r_{\mathrm{ext}}^{2} \log \left(r_{\mathrm{ext}}\right)-3}{4\left(r_{\mathrm{ext}}^{2}-1\right)^{2}}\right]+\frac{2 \gamma q_{\mathrm{ext}}}{r_{\mathrm{ext}}^{2}-1} .
\end{aligned}
$$

Substituting Eq. (A.29) into Eq. (24d) and using the previous closed formulas, the pressure is

$$
\begin{aligned}
h_{2}(r, t) & =\frac{\pi^{2}}{2} \sum_{i=1}^{\infty} \frac{k_{i}^{2} \tilde{g}\left(k_{i}, t\right) \mathcal{I}_{1,0}\left(k_{i}, 1, r\right) J_{1}^{2}\left(r_{\mathrm{ext}} k_{i}\right)}{J_{1}^{2}\left(k_{i}\right)-J_{1}^{2}\left(r_{\mathrm{ext}} k_{i}\right)}+\frac{2}{r_{\mathrm{ext}}^{2}-1}\left(\frac{r^{2}}{4}+t\right)-\frac{r_{\mathrm{ext}}^{2}}{r_{\mathrm{ext}}^{2}-1} \log (r) \\
& -\frac{3 r_{\mathrm{ext}}^{4}-4 r_{\mathrm{ext}}^{4} \log \left(r_{\mathrm{ext}}\right)-2 r_{\mathrm{ext}}^{2}-1}{4\left(r_{\mathrm{ext}}^{2}-1\right)^{2}}+q_{\mathrm{ext}}\left[\frac{2}{r_{\mathrm{ext}}^{2}-1}\left(\frac{r^{2}}{4}+t\right)-\frac{\log (r)}{r_{\mathrm{ext}}^{2}-1}\right. \\
& \left.-\frac{r_{\mathrm{ext}}^{4}+2 r_{\mathrm{ext}}^{2}-4 r_{\mathrm{ext}}^{2} \log \left(r_{\mathrm{ext}}\right)-3}{4\left(r_{\mathrm{ext}}^{2}-1\right)^{2}}\right]+\frac{2 \gamma q_{\mathrm{ext}}\left(1-\mathrm{e}^{-t / \gamma}\right)}{r_{\mathrm{ext}}^{2}-1},
\end{aligned}
$$

where

$$
\begin{aligned}
\tilde{g}\left(k_{i}, t\right) & =\tilde{\chi}\left(k_{i}, t\right)+\left(q_{\mathrm{ext}} / r_{\mathrm{ext}}\right) \tilde{\mathcal{G}}\left(k_{i}\right)\left[\tilde{Q}_{2}\left(k_{i}, t\right)-\tilde{R}_{1}\left(k_{i}, t\right)\right] \\
\tilde{\chi}\left(k_{i}, t\right) & =\frac{1}{\pi k_{i}^{3} v} \exp \left\{-\frac{(\xi+v)}{2 \psi} t\right\}\left[\left(\exp \left\{\frac{v}{\psi} t\right\}-1\right) \varrho-\left(\exp \left\{\frac{v}{\psi} t\right\}+1\right) \nu\right], \\
\tilde{R}_{1}\left(k_{i}, t\right) & =\frac{\pi}{2} k_{i} \tilde{\chi}\left(k_{i}, t\right),
\end{aligned}
$$


and $k_{i}$ are the positive roots of $\mathcal{I}_{1,1}\left(k_{i}, 1, r_{\text {ext }}\right)$. Note that to find $\tilde{g}\left(k_{i}, t\right)$, we write $\tilde{Q}_{1}\left(k_{i}, t\right)=1 / k_{i}^{2}+\tilde{R}_{1}\left(k_{i}, t\right)$.

The time-dependent terms in Eqs. (A.30) and (A.31) are included in Eq. (A.35) in order to describe the long-time fluid behavior. It is worth mentioning that these terms are omitted in the Cinelli formulas (Cinelli, 1965) of the NNBCs case. Equation (A.35) recovers the results in Muskat (1934), Matthews and Russell (1967), and del Angel et al. (2014) when the limit of single-porosity is taken. 